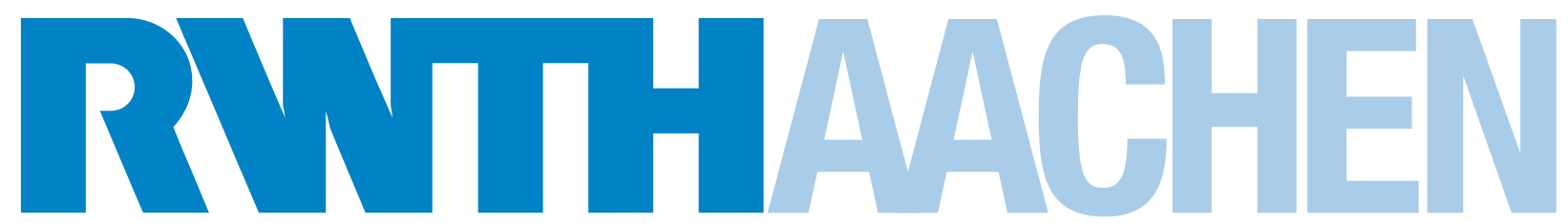

RHEINISCH-WESTFÄLISCHE TECHNISCHE HOCHSCHULE AACHEN Institut für Mathematik

Tangency properties of sets with finite geometric curvature energies

by

Sebastian Scholtes

Report No. 52

February

2012

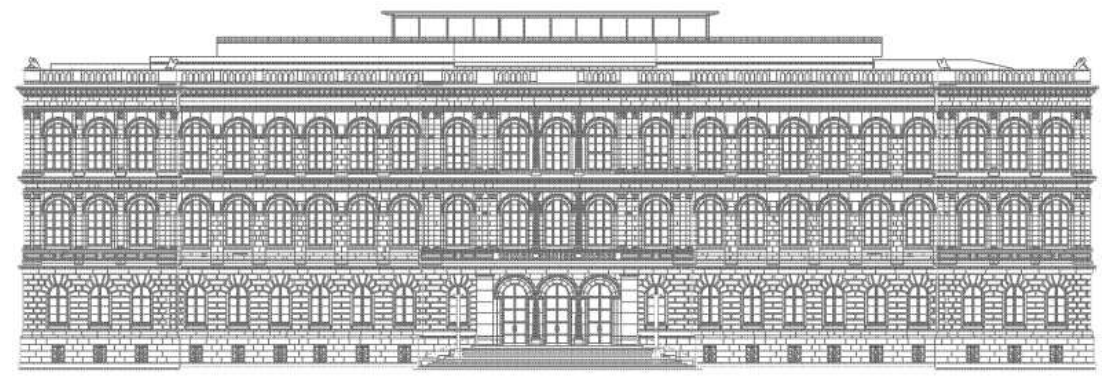

Institute for Mathematics, RWTH Aachen University

Templergraben 55, D-52062 Aachen

Germany 


\title{
Tangency properties of sets with finite geometric curvature energies
}

\author{
Sebastian Scholtes
}

April 3, 2012

\begin{abstract}
We investigate inverse thickness $1 / \Delta$ and the integral Menger curvature energies $\mathcal{U}_{p}^{\alpha}, \mathcal{I}_{p}^{\alpha}$ and $\mathcal{M}_{p}^{\alpha}$, to find that finite $1 / \Delta$ or $\mathcal{U}_{p}^{\alpha}$ implies the existence of an approximate $\alpha$-tangent at all points of the set, when $p \geq \alpha$ and that finite $\mathcal{I}_{p}^{\alpha}$ or $\mathcal{M}_{p}^{\alpha}$ implies the existence of a weak approximate $\alpha$-tangent at every point of the set for $p \geq 2 \alpha$ or $p \geq 3 \alpha$, respectively, if some additional density properties hold. This includes the scale invariant case $p=2$ for $\mathcal{I}_{p}^{1}$ and $p=3$ for $\mathcal{M}_{p}^{1}$, for which, to the best of our knowledge, no regularity properties are established up to now. Furthermore we prove that for $\alpha=1$ these exponents are sharp, i.e., that if $p$ lies below the threshold value of scale innvariance, then there exists a set containing points with no (weak) approximate 1-tangent, but such that the corresponding energy is still finite. For $\mathcal{I}_{p}^{1}$ and $\mathcal{M}_{p}^{1}$ we give an example of a set which possesses a point that has no approximate 1-tangent, but finite energy for all $p \in(0, \infty)$ and thus show that the existence of weak approximate 1-tangents is the most we can expect, in other words our results are also optimal in this respect.
\end{abstract}

Mathematics Subject Classification (2000): 28A75

\section{Introduction}

In [Lég99] J. C. Léger was able to show a remarkable theorem ${ }^{1}$, which states that onedimensional Borel sets in $\mathbb{R}^{n}$ with finite integral Menger curvature $\mathcal{M}_{2}^{1}$ are 1-rectifiable. Here, integral Menger ${ }^{2}$ curvature of a set $X \subset \mathbb{R}^{n}$ refers to the triple integral over the squared inverse circumradius ${ }^{3}$, i.e.

$$
\mathcal{M}_{p}^{\alpha}(X):=\int_{X} \int_{X} \int_{X}[r(x, y, z)]^{-p} \mathrm{~d} \mathcal{H}^{\alpha}(x) \mathrm{d} \mathcal{H}^{\alpha}(y) \mathrm{d} \mathcal{H}^{\alpha}(z)
$$

for $p=2$ and $\alpha=1$. The circumradius $r(x, y, z)$ is the radius of the unique circle on which the vertices of the non-degenerate triangle $\{x, y, z\}$ lie; in case of a degenerate triangle it is set to be infinite. These results for $\mathcal{M}_{2}^{1}$ were later extended to metric spaces in [Hah08],

\footnotetext{
${ }^{1}$ Léger refers to an unplublished work of G. David that had inspired his work and that he took as a guideline for the proof.

${ }^{2}$ Named after Karl Menger, because in [Men30] Menger introduced the limit of the inverse circumradius, when the three points in the argument converge to a single point, as a pointwise curvature.

${ }^{3}$ For other applications of the circumradius see [Sch12].
} 
and in [LM01] to sets of fractional dimension, where $C^{1}-\alpha$-rectifiability of measurable sets with positive and finite $\mathcal{H}^{\alpha}$ measure could be shown if $\mathcal{M}_{2 \alpha}^{\alpha}$ is finite and $\alpha \in(0,1 / 2]$ under the additional assumption that these sets are $\alpha$-Ahlfors regular ${ }^{4}$. As a consequence Léger's theorem also ensures that an $\mathcal{H}^{1}$ measurable set $E \subset \mathbb{R}^{n}$ with $\mathcal{M}_{2}^{1}(E)<\infty$ has approximate 1 -tangents at $\mathcal{H}^{1}$ a.e point. By an approximate $\alpha$-tangent at a point $x$ we mean a direction $s \in \mathbb{S}^{n-1}$, such that

$$
\lim _{r \downarrow 0} \frac{\mathcal{H}^{\alpha}\left(\left[X \backslash C_{s, \varepsilon}(x)\right] \cap \bar{B}_{r}(x)\right)}{(2 r)^{\alpha}}=0 \quad \text { for all } \varepsilon>0,
$$

where $C_{s, \varepsilon}(x)$ is the double cone with opening angle $\varepsilon$ in direction $s$ about $x$, cf. [Mat95, p.203]; for different tangential regularity properties compare also to [MM88]. One might think of it as a kind of geometric or measure theoretic counterpart to differentiability. Roughly speaking it means that the set is locally well approximated by the approximate tangent. For example a regular, differentiable curve has approximate 1-tangents at all points and these tangents coincide with the usual tangent, but the arc length parametrisation of the set $S:=\{(x, 0) \mid x \in[0,1]\} \cup\left\{\left(x, x^{2}\right) \mid x \in[0,1]\right\}$ has no tangent at $(0,0)$, despite the set having an approximate 1-tangent at this point, see Remark 4.6.

Now one could ask if the condition $\mathcal{M}_{2}^{1}(X)<\infty$ also guarantees that the set has approximate 1-tangents at all points, or, if this is not the case, which influence, if any, the exponent $p$ of the energy $\mathcal{M}_{p}^{1}$ has on these matters. This question and related topics are the subject of this paper.

Complementary to this research, where highly irregular sets are permitted, was the investigation of rectifiable curves, which have a classic tangent $\mathcal{H}^{1}$ a.e. to begin with, of finite $\mathcal{M}_{p}^{1}$ energy. It turns out, see [SSvdM10], that for $p>3$ this guarantees that the curve is simple and that the arc length parametrisation is of class $C^{1,1-3 / p}$, which can be interpreted as a geometric Morrey-Sobolev imbedding. In [Bla11a] it could be shown that the space of curves with finite $\mathcal{M}_{p}^{1}$ for $p>3$ is that of Sobolev Slobodeckij embeddings of class $W^{2-2 / p, p}$. The same program has also been conducted for a different kind of energy, the so-called tangent point energy in [SvdM12b, Bla11b].

We would like to point out the important role of integral Menger curvature for $p=2$ in the solution of the Painlevé problem i.e. to find geometric characterisations of removable sets for bounded analytic functions, see [Paj02, Dud10] for a detailed presentation and references.

Besides integral Menger curvature there are other interesting curvature energies that have been investigated in the same vein. In [GM99] Gonzales and Maddocks proposed their notion of thickness

$$
\Delta[X]:=\inf _{\substack{x, y, z \in X \\ x \neq y \neq z \neq x}} r(x, y, z)
$$

of a knot $X$, which is the infimum of the circumradius $r(x, y, z)$ over all triangles $\{x, y, z\}$

\footnotetext{
${ }^{4}$ It was also shown that these results are sharp, i.e. wrong for $s \in(1 / 2,1)$, but that there is no hope of maintaining these results for $s \in(0,1)$ if one drops the $\alpha$-Ahlfors regularity.
} 
on the curve, and also encouraged to investigate different integral curvature energies

$$
\begin{aligned}
\mathcal{U}_{p}^{\alpha}(X) & :=\int_{X}\left[\inf _{\substack{y, z \in X \\
x \neq y \neq z \neq x}} r(x, y, z)\right]^{-p} \mathrm{~d} \mathcal{H}^{\alpha}(x), \\
\mathcal{I}_{p}^{\alpha}(X) & :=\int_{X} \int_{X}\left[\inf _{\substack{z \in X \\
x \neq y \neq z \neq x}} r(x, y, z)\right]^{-p} \mathrm{~d} \mathcal{H}^{\alpha}(x) \mathrm{d} \mathcal{H}^{\alpha}(y),
\end{aligned}
$$

and $\mathcal{M}_{p}^{\alpha}$, where the inverse circumradius is integrated to the power $p$ and the infimisations are successively replaced by integrations. That arc length parametrisations of curves with finite inverse thickness are actually of class $C^{1,1}$ and the existence of ideal knots, which are minimizers of the inverse thickness in a knot class under the restriction of fixed length, was shown in [GMSvdM02, CKS02, GdlL03]; for further research in this direction see also [SvdM03, SvdM04]. In the series of works [SvdM07, SSvdM09, SSvdM10] the integral curvature energies $\mathcal{U}_{p}^{1}, \mathcal{I}_{p}^{1}$ and $\mathcal{M}_{p}^{1}$ have been investigated for closed rectifiable curves, to find that the arc length parametrisations of curves with finite energy for $p \in[1, \infty)$, $p \in(2, \infty)$ and $p \in(3, \infty)$, respectively, are simple and actually belong to the class $C^{1, \beta_{\mathcal{F}}(p)}$, where $\beta_{\mathcal{U}}(p)=1-1 / p, \beta_{\mathcal{I}}(p)=1-2 / p$ and $\beta_{\mathcal{M}}(p)=1-3 / p$. In [Bla11a] it could be shown that the space of curves with finite $\mathcal{I}_{p}^{1}$ for $p>2$ and $\mathcal{M}_{p}^{1}$ for $p>3$ is that of Sobolev Slobodeckij embeddings of class $W^{2-1 / p, p}$ and $W^{2-2 / p, p}$, respectively. Similar kind of energies for surfaces and higher dimensional sets have been examined in [SvdM05, SvdM06, SvdM11, LW09, LW11, Kol11, KS11, SvdM12a, BK12].

As mentioned in the very beginning the purpose of this paper is to investigate which pointwise tangential properties can be expected of sets in Euclidean space with finite energy. To be more precise we will investigate if a set $X$ possesses an approximate $\alpha$ tangent or at least a weak approximate $\alpha$-tangent at every point $x$. A weak approximate $\alpha$-tangent is a mapping $s:(0, \rho) \rightarrow \mathbb{S}^{n-1}$, such that

$$
\lim _{r \downarrow 0} \frac{\mathcal{H}^{\alpha}\left(\left[X \backslash C_{s(r), \varepsilon}(x)\right] \cap \bar{B}_{r}(x)\right)}{(2 r)^{\alpha}}=0 \quad \text { for all } \varepsilon>0 .
$$

For the example of the T-shaped set $E:=([-1,1] \times\{0\}) \cup(\{0\} \times[0,1])$ it is shown that $\mathcal{M}_{2}^{1}(E)<\infty$ does not suffice to infer that the set has weak approximate 1-tangents at all points with positive lower density, see Lemma 8.1. So it seems that these properties might depend on the exponent $p$ and the parameter $\alpha$ of the integral curvature energies $\mathcal{U}_{p}^{\alpha}, \mathcal{I}_{p}^{\alpha}$ and $\mathcal{M}_{p}^{\alpha}$. Thus our aim is to find conditions on $p$ and $\alpha$ that ensure the existence of $\alpha$ tangents at all points with positive lower density. We shall solve this question thoroughly, to be honest with one minor additional technical requirement in case of $\mathcal{M}_{p}^{\alpha}$, namely $\Theta^{* \alpha}\left(\mathcal{H}^{\alpha}, X, x\right)<\infty$, that, despite our best efforts, we were not able to remove. We have gathered the findings from different sections of the present paper in the following Theorem. Note that compared to [LM01] we do not require the set to be measurable and $\alpha$-Ahlfors regular and have more detailed information on which points do possess tangents, but we pay for that by a more restrictive requirement on the parameter $p$. We also want to remark that in [Lin97, 1.5 Corollary, p.13] it is shown that for $\alpha>1$ and an $\mathcal{H}^{\alpha}$ measurable set $X \subset \mathbb{R}^{n}$ with $0<\mathcal{H}^{\alpha}(X)<\infty$ we always have $\mathcal{M}_{2 \alpha}^{\alpha}(X)=\infty$, which somewhat restricts the extent of the next theorem for $\alpha>1$. On the other hand, however, there are a lot more sets allowed in the theorem that still could have finite $\mathcal{M}_{2 \alpha}^{\alpha}$. 
Theorem 1.1 (Main result).

Let $X \subset \mathbb{R}^{n}, x \in \mathbb{R}^{n}, \alpha \in(0, \infty)$.

- Let $1 / \Delta[X]<\infty, \mathcal{H}^{1}(X)<\infty$. Then $X$ has an approximate 1 -tangent at $x$.

- Let $p \in[\alpha, \infty), \mathcal{U}_{p}^{\alpha}(X)<\infty$. Then $X$ has an approximate $\alpha$-tangent at $x$.

- Let $p \in[2 \alpha, \infty), \mathcal{I}_{p}^{\alpha}(X)<\infty$ and $\Theta_{*}^{\alpha}\left(\mathcal{H}^{\alpha}, X, x\right)>0$. Then $X$ has a weak approximate $\alpha$-tangent at $x$.

- Let $p \in[3 \alpha, \infty), \mathcal{M}_{p}^{\alpha}(X)<\infty$ and $0<\Theta_{*}^{\alpha}\left(\mathcal{H}^{\alpha}, X, x\right) \leq \Theta^{* \alpha}\left(\mathcal{H}^{\alpha}, X, x\right)<\infty$. Then $X$ has a weak approximate $\alpha$-tangent at $x$.

To the best of our knowledge these are the first results regarding regularity that incorporate the critical cases $p=2$ for $\mathcal{I}_{p}^{1}$ and $p=3$ for $\mathcal{M}_{p}^{1}$. Moreover, we show that the exponents are sharp for $\alpha=1$, that is, there is a set, namely the T-shaped set $E$ from above, that contains a point without weak approximate 1-tangent and has finite energy if $p$ is below the respective threshold value.

Proposition 1.2 (Exponents are sharp for $\alpha=1$ ).

For $E:=([-1,1] \times\{0\}) \cup(\{0\} \times[0,1])$ we have

- $\mathcal{U}_{p}^{1}(E)<\infty$ for $p \in(0,1)$,

- $\mathcal{I}_{p}^{1}(E)<\infty$ for $p \in(0,2)$,

- $\mathcal{M}_{p}^{1}(E)<\infty$ for $p \in(0,3)$.

Furthermore we demonstrate that there is a set $F$ that has a point without an approximate 1 -tangent and finite $\mathcal{I}_{p}^{1}$ and $\mathcal{M}_{p}^{1}$ for all $p \in(0, \infty)$. Hence there is no hope of obtaining the main result for approximate 1-tangents instead of weak approximate 1-tangents for these two energies.

Proposition 1.3 (Weak approximate 1-tangents are optimal for $\alpha=1$ ). There is a set $F, x \in \mathbb{R}^{n}$, such that $F$ has no approximate 1-tangent at $x$ and

- $\mathcal{I}_{p}^{1}(F)<\infty$ for $p \in(0, \infty)$,

- $\mathcal{M}_{p}^{1}(F)<\infty$ for $p \in(0, \infty)$.

To put these results into perspective, we give some simple examples of what they infer, in case of the integral Menger curvature $\mathcal{M}_{p}^{1}$. For a curve and $p \geq 3$ we only obtain weak approximate 1-tangents, which is hardly a new result, except for the case $p=3$, as we already knew for $p>3$ that these curves are actually in $W^{2-2 / p, p}$ by [SSvdM10, Bla11a], but now we also know that it is possible for more general connected compact objects to have finite integral Menger curvature for $p \in(0,3)$, objects that cannot be parametrised by a simple curve, like the set $E$. On the other hand there are objects with infinite energy, which for instance are constructed by the following principle: let $X$ be a set, $x \in X$ with positive density and an approximate 1-tangent $s$ at $x$, further let $\varphi_{A}(X)$ be the set $X$ rotated by a rotation matrix $A$ about the point $x$ in such a way that the axis of rotation does not coincide with $s$. Then the set $X \cup \varphi_{A}(X)$ - for example a polygon with two edges - has no weak approximate 1 -tangent at $x$, see Lemma 4.11 , and hence infinite $\mathcal{M}_{p}^{1}$ for 
$p \geq 3$. Here previously no conclusive statement was possible. In addition, for $p \in(0,3)$ all polygons have finite $\mathcal{M}_{p}^{1}$ energy, which can be seen using techniques of this paper. Similar statements hold for $\mathcal{U}_{p}^{1}$ and $\mathcal{I}_{p}^{1}$ for $p$ below the scale invariant threshold value; see [Sch11].

The paper is organised as follows: Section 2 introduces integral curvature energies for arbitrary metric spaces, as this is no more complicated than doing so for arbitrary sets in $\mathbb{R}^{n}$ and even provides a simpler notation. Then, in Section 3, we give lower bounds for the Hausdorff measure of annuli under certain conditions on the Hausdorff density. We also introduce a new and slightly wider notion of Hausdorff density for set valued mappings. In Section 4 we give some examples and simple properties of the different notions of tangents. Finally we are ready to prove the main theorem and compute the energies $1 / \Delta \& \mathcal{U}_{p}^{\alpha}, \mathcal{I}_{p}^{\alpha}$ and $\mathcal{M}_{p}^{\alpha}$ of the set $E$ in the Sections 5, 6 and 7, respectively. The topic of Section 8 is the proof of Proposition 1.3. To improve readability we have deferred several technical issues to the appendix.

\section{Acknowledgements}

The author wishes to thank his advisor Heiko von der Mosel for constant support and encouragement, reading and discussing the present paper, as well as giving many helpful remarks, like the idea to allow for $\alpha \neq 1$ as in [LM01]. He is also thankful to Martin Meurer for the joint efforts that lead to Lemma 2.14. Furthermore the author is indebted to Thomas El Khatib, who gave some helpful remarks and a better proof for Lemma C.1.

\section{Curvature energies and notation}

For a set $X$ with outer measure $\mathcal{V}$ we write $\mathcal{C}(\mathcal{V})$ for the $\mathcal{V}$ measurable sets of $X$, i.e. those sets $E$, which are measurable in the sense of Carathéory:

$$
\mathcal{V}(M)=\mathcal{V}(M \cap E)+\mathcal{V}(M \backslash E) \text { for all } M \subset X .
$$

Let $(X, \tau)$ be a topological space - in this paper the topology is always induced by a metric - then $\mathcal{B}(X)$ denotes the Borel sets of $(X, \tau)$. For two measurable spaces $(X, \mathcal{A})$ and $(Y, \mathcal{B})$ we say that a function $f:(X, \mathcal{A}) \rightarrow(Y, \mathcal{B})$ is $\mathcal{A}-\mathcal{B}$ measurable, if $f^{-1}(B) \in \mathcal{A}$ for all $B \in \mathcal{B}$. By $\mathcal{H}^{\alpha}$ we denote the $\alpha$-dimensional Hausdorff measure on a metric space $(X, d)$ and by $\mathcal{L}^{n}$ the $n$-dimensional Lebesgue measure on $\mathbb{R}^{n}$. The extended real numbers are indicated by the symbol $\overline{\mathbb{R}}$.

The thickness of a set was introduced by O. Gonzales and J. Maddocks in [GM99], where they also suggested to investigate the integral curvature energies $\mathcal{U}_{p}^{1}, \mathcal{I}_{p}^{1}$ and $\mathcal{M}_{p}^{1}$, which will be defined subsequently.

Definition 2.1 (Circumradius, interm. and global radius of curv., thickness). Let $(X, d)$ be a metric space. We define the circumradius of three distinct points $x, y, z \in$ $X$ as the circumradius of the triangle defined by the, up to Euclidean motions unique, isometric embedding of these three points in the Euclidean plane, i.e.

$$
\begin{aligned}
r:\{(x, y, z) & \left.\in X^{3} \mid d(x, y), d(y, z), d(z, x)>0\right\}=: D \rightarrow \overline{\mathbb{R}}, \\
(x, y, z) & \mapsto \frac{a b c}{\sqrt{(a+b+c)(a+b-c)(a-b+c)(-a+b+c)}},
\end{aligned}
$$


where $a:=d(x, y), b:=d(y, z), c:=d(z, x)$ and $\alpha / 0=\infty$ for any $\alpha>0$. We also write $X_{0}:=X^{3} \backslash D$. Now we define the mappings $\rho: X^{2} \backslash \operatorname{diag}(X) \rightarrow \overline{\mathbb{R}}$ and $\rho_{G}: X \rightarrow \overline{\mathbb{R}}$ by

$$
\rho(x, y):=\inf _{\substack{w \in X \\ x \neq y \neq w \neq x}} r(x, y, w) \text { and } \rho_{G}(x):=\inf _{\substack{v, w \in X \\ x \neq v \neq w \neq x}} r(x, v, w)
$$

which are often called intermediate and global radius of curvature, respectively. Here $\operatorname{diag}(X):=\{(x, x) \mid x \in X\}$ denotes the diagonal of $X$. The thickness is then defined to be

$$
\Delta[X]:=\inf _{\substack{u, v, w \in X \\ u \neq v \neq w \neq u}} r(u, v, w)
$$

Remark 2.2 (Different formulas for the circumradius).

We note that in $\mathbb{R}^{n}$ there are various formulas for the circumradius, for example one has the following representations for $x, y, z \in \mathbb{R}^{n}$ mutually distinct [Paj02, (14) and (15), p.29]

$$
r(x, y, z)=\frac{|x-y|}{2|\sin (\measuredangle(x, z, y))|}=\frac{|x-z||y-z|}{2 \operatorname{dist}\left(z, L_{x, y}\right)},
$$

where $L_{x, y}:=x+\mathbb{R}(x-y)$ is the straight line connecting $x$ and $y$.

Lemma 2.3 (Various curvature radii are upper semi-continuous). Let $(X, d)$ be a metric space. Then

$$
\begin{array}{rll}
r: X^{3} \backslash X_{0} & \rightarrow \overline{\mathbb{R}} & \text { is continuous } \\
\rho: X^{2} \backslash \operatorname{diag}(X) & \rightarrow \overline{\mathbb{R}} & \text { is upper semi-continuous } \\
\rho_{G}: \quad X \quad & \rightarrow \overline{\mathbb{R}} & \text { is upper semi-continuous. }
\end{array}
$$

Proof. Step 1 Let $\left(\left(x_{n}, y_{n}, z_{n}\right)\right)_{n \in \mathbb{N}} \subset D$ and $(x, y, z) \in D$ such that $\left(x_{n}, y_{n}, z_{n}\right) \rightarrow(x, y, z)$ in $X^{3}$ and set $f(x, y, z):=(-a+b+c)(a-b+c)(a+b-c)$.

Case 1 Let us first assume that $f(x, y, z) \neq 0$. Then $f(x, y, z)>0$ and as $(x, y, z) \in D$ we have $r(x, y, z)<\infty$. Since $f$ is continuous, see Lemma A.1 we have $f\left(x_{n}, y_{n}, z_{n}\right) \geq$ $f(x, y, z) / 2$ for $n$ large enough. Therefore $r\left(x_{n}, y_{n}, z_{n}\right) \rightarrow r(x, y, z)$, because the numerator of (1) is also continuous.

Case 2 If on the other hand $f(x, y, z)=0$, we have $f\left(x_{n}, y_{n}, z_{n}\right) \rightarrow 0$ and $g\left(x_{n}, y_{n}, z_{n}\right):=$ $d\left(x_{n}, y_{n}\right) d\left(y_{n}, z_{n}\right) d\left(z_{n}, x_{n}\right)>g(x, y, z) / 2$ for $n$ large enough, which gives us $r\left(x_{n}, y_{n}, z_{n}\right) \rightarrow$ $r(x, y, z)=\infty$.

Step 2 If we set $f_{z}:(x, y) \mapsto r(x, y, z)$ then according to the previous item the functions $f_{z}$ are upper semi-continuous and therefore, see [Bra02, Remark 1.4 (ii), p.21], also is

$$
\rho(x, y)=\inf _{z \in X \backslash\{x, y\}} f_{z}(x, y)
$$

Step 3 By arguing analogous to the proof of the preceding item we have that

$$
\rho_{G}(x)=\inf _{y \in X \backslash\{x\}} \rho(x, y)
$$

is upper semi-continuous. 
Lemma 2.4 (Reciprocal radii of curvature are l.s.c. and measurable). Let $(X, d)$ be a metric space. Then the functions

$$
\begin{aligned}
\kappa_{G}: X \rightarrow \overline{\mathbb{R}}, & x \mapsto \frac{1}{\rho_{G}(x)}, \\
\kappa_{i}: X^{2} \rightarrow \overline{\mathbb{R}}, \quad(x, y) & \mapsto \begin{cases}\frac{1}{\rho(x, y)}, & (x, y) \in X^{2} \backslash \operatorname{diag}(X), \\
0, & \text { else, }\end{cases} \\
\kappa: X^{3} \rightarrow \overline{\mathbb{R}},(x, y, z) & \mapsto \begin{cases}\frac{1}{r(x, y, z)}, & (x, y, z) \in X^{3} \backslash X_{0}, \\
0, & \text { else, }\end{cases}
\end{aligned}
$$

with the convention $1 / 0=\infty$ and $1 / \infty=0$ are lower semi-continuous and $\mathcal{B}(X)-\mathcal{B}(\overline{\mathbb{R}})$, $\mathcal{B}\left(X^{2}\right)-\mathcal{B}(\overline{\mathbb{R}})$ and $\mathcal{B}\left(X^{3}\right)-\mathcal{B}(\overline{\mathbb{R}})$ measurable, respectively.

Proof. Considering Lemmata 2.3 and A.2 the functions $\kappa_{G}, \kappa_{i}$ and $\kappa$ are lower semicontinuous on $X, X^{2} \backslash \operatorname{diag}(X)$ and $X^{3} \backslash X_{0}$ respectively. This proves the proposition for $\kappa_{G}$. Now considering that the excluded sets $\operatorname{diag}(X)$ and $X_{0}$ are closed, Lemma A.7, and that the functions are non-negative on the whole space and 0 on these sets, we know that they are lower semi-continuous on the entire space by Lemma A.6. Now Lemma A.3 gives us Borel measurability.

\section{Definition 2.5 (A menagerie of integral curvature energies).}

Let $(X, d)$ be a metric space and $\alpha, p \in(0, \infty)$. We are now able to define the following two-parameter families of integral curvature energies

$$
\begin{aligned}
\mathcal{U}_{p}^{\alpha}(X) & :=\int_{X} \kappa_{G}^{p}(x) \mathrm{d} \mathcal{H}^{\alpha}(x), \\
\mathcal{I}_{p}^{\alpha}(X) & :=\int_{X} \int_{X} \kappa_{i}^{p}(x, y) \mathrm{d} \mathcal{H}^{\alpha}(x) \mathrm{d} \mathcal{H}^{\alpha}(y), \\
\mathcal{M}_{p}^{\alpha}(X) & :=\int_{X} \int_{X} \int_{X} \kappa^{p}(x, y, z) \mathrm{d} \mathcal{H}^{\alpha}(x) \mathrm{d} \mathcal{H}^{\alpha}(y) \mathrm{d} \mathcal{H}^{\alpha}(z) .
\end{aligned}
$$

The last of these energies, $\mathcal{M}_{p}^{\alpha}$, is often called $\alpha$-dimensional (integral) $p$-Menger curvature.

Remark 2.6 (Subtle differences in possible definitions of energies).

We want to remark that in the Euclidean case the measure in the integrals is the Hausdorff measure on the set $X$ (respective to the subspace metric, i.e. the restriction of the metric of $\mathbb{R}^{n}$ to the set $X$ ), in contrast to the Hausdorff measure on $\mathbb{R}^{n}$. As we shall see shortly this enables us to include non-measurable sets, contrary to the other approach, where the energy might not exist on non-measurable sets, which can easily be seen by the example of a Vitali type set on the unit circle. We suspect that the gain of permitted sets when comparing [Hah08] for $\mathbb{R}^{n}$ to [Lég99], where only Borel sets were permitted, might be related to this matter.

We shall now be concerned with the existence of these integral curvature energies, which is why we first take a closer look at the integrands. 
Lemma 2.7 (Various integrand functions are l.s.c. and measurable). Let $(X, d)$ be a metric space. Then for all $p \in(0, \infty)$ the following functions

$$
\begin{aligned}
y & \mapsto \int_{X} \kappa_{i}^{p}(x, y) d \mathcal{H}^{\alpha}(x) \\
y & \mapsto \int_{X} \kappa^{p}(x, y, z) d \mathcal{H}^{\alpha}(x) \quad \text { for all } z \in X \\
z & \mapsto \int_{X} \int_{X} \kappa^{p}(x, y, z) d \mathcal{H}^{\alpha}(x) d \mathcal{H}^{\alpha}(y)
\end{aligned}
$$

are lower semi-continuous and $\mathcal{B}(X)-\mathcal{B}(\overline{\mathbb{R}})$ measurable.

Proof. Step 1 By Lemma 2.4, $\kappa \geq 0$ and Lemma A.4 we know that $\kappa^{p}$ is lower semicontinuous. Let $a_{n} \rightarrow a$ in $X$. As for fixed $x, y, z \in X$ we have $\left(a_{n}, y, z\right) \rightarrow(a, y, z)$ and therefore

$$
\kappa^{p}(a, y, z) \leq \liminf _{n \rightarrow \infty} \kappa^{p}\left(a_{n}, y, z\right),
$$

so that $\kappa^{p}(\cdot, y, z), \kappa^{p}(x, \cdot, z)$ and $\kappa^{p}(x, y, \cdot)$ are lower semi-continuous and hence $\mathcal{B}(X)$ $\mathcal{B}(\overline{\mathbb{R}})$ measurable, see Lemma A.3. Using Fatou's Lemma [EG92, Theorem 1, p.19] we obtain

$$
\int_{X} \kappa^{p}(x, y, a) \mathrm{d} \mathcal{H}^{\alpha}(x) \leq \int_{X} \liminf _{n \rightarrow \infty} \kappa^{p}\left(x, y, a_{n}\right) \mathrm{d} \mathcal{H}^{\alpha}(x) \leq \liminf _{n \rightarrow \infty} \int_{X} \kappa^{p}\left(x, y, a_{n}\right) \mathrm{d} \mathcal{H}^{\alpha}(x) .
$$

This tells us that for fixed $x, y, z$ the mappings $\int_{X} \kappa^{p}(x, \cdot, z) \mathrm{d} \mathcal{H}^{\alpha}(x)$ and $\int_{X} \kappa^{p}(x, y, \cdot) \mathrm{d} \mathcal{H}^{\alpha}(x)$ are lower semi-continuous and hence measurable.

Step 2 Let $z_{n} \rightarrow z$ in $X$. If we use Fatou's Lemma and integrate again, we obtain

$$
\begin{aligned}
& \int_{X} \int_{X} \kappa^{p}(x, y, z) \mathrm{d} \mathcal{H}^{\alpha}(x) \mathrm{d} \mathcal{H}^{\alpha}(y) \leq \int_{X} \liminf _{n \rightarrow \infty} \int_{X} \kappa^{p}\left(x, y, z_{n}\right) \mathrm{d} \mathcal{H}^{\alpha}(x) \mathrm{d} \mathcal{H}^{\alpha}(y) \\
& \leq \liminf _{n \rightarrow \infty} \int_{X} \int_{X} \kappa^{p}\left(x, y, z_{n}\right) \mathrm{d} \mathcal{H}^{\alpha}(x) \mathrm{d} \mathcal{H}^{\alpha}(y),
\end{aligned}
$$

so that $z \mapsto \int_{X} \int_{X} \kappa^{p}(x, y, z) \mathrm{d} \mathcal{H}^{\alpha}(x) \mathrm{d} \mathcal{H}^{\alpha}(y)$ is lower semi-continuous and hence measurable. For the function involving $\kappa_{i}$ we argue analogously.

Lemma 2.8 (Integral curvature energies are well-defined).

Let $(X, d)$ be a metric space. Then for all $\alpha, p \in(0, \infty)$ the curvature energies $\mathcal{U}_{p}^{\alpha}(X)$, $\mathcal{I}_{p}^{\alpha}(X)$ and $\mathcal{M}_{p}^{\alpha}(X)$ are well defined.

Proof. This is a simple consequence of Lemma 2.4 and Lemma 2.7 together with the fact that the integrands are non-negative, see [EG92, Remark, p.18].

Lemma 2.9 (Inequality between integral curvature energies).

Let $(X, d)$ be a metric space with $\mathcal{H}^{\alpha}(X)<\infty$ and $\alpha, p \in(0, \infty)$, then

$$
\mathcal{M}_{p}^{\alpha}(X) \leq \mathcal{H}^{\alpha}(X) \mathcal{I}_{p}^{\alpha}(X) \leq \mathcal{H}^{\alpha}(X)^{2} \mathcal{U}_{p}^{\alpha}(X) \leq \frac{\mathcal{H}^{\alpha}(X)^{3}}{\Delta[X]^{p}}
$$


Proof. Clearly for all distinct $x, y, z \in X$ we have

$$
\Delta[X] \leq \rho_{G}(x) \leq \rho(x, y) \leq r(x, y, z)
$$

which gives us

$$
\kappa(x, y, z) \leq \kappa_{i}(x, y) \leq \kappa_{G}(x) \leq \frac{1}{\Delta[X]} \text { for all } x, y, z \in X
$$

and thus the proposition.

By successively using the Hölder inequality from the inner to the outer integral one can easily prove

Lemma 2.10 (Comparison of curvature energies for different $p$ ).

Let $(X, d)$ be a metric space with $\mathcal{H}^{\alpha}(X)<\infty, \alpha \in(0, \infty)$ and $0<p<q<\infty$. Then

$$
\begin{aligned}
\mathcal{U}_{p}^{\alpha}(X) & \leq \mathcal{H}^{\alpha}(X)^{(1-p / q)} \mathcal{U}_{q}^{\alpha}(X)^{p / q} \\
\mathcal{I}_{p}(X)^{\alpha} & \leq \mathcal{H}^{\alpha}(X)^{2(1-p / q)} \mathcal{I}_{q}^{\alpha}(X)^{p / q} \\
\mathcal{M}_{p}^{\alpha}(X) & \leq \mathcal{H}^{\alpha}(X)^{3(1-p / q)} \mathcal{M}_{q}^{\alpha}(X)^{p / q}
\end{aligned}
$$

Proof. For $a=q / p>1$ and $b=q /(q-p)$ we obtain

$$
\begin{aligned}
\mathcal{M}_{p}^{\alpha}(X) & =\int_{X} \int_{X} \int_{X} \kappa^{p}(x, y, z) \mathrm{d} \mathcal{H}^{\alpha}(x) \mathrm{d} \mathcal{H}^{\alpha}(y) \mathrm{d} \mathcal{H}^{\alpha}(z) \\
\leq & \int_{X} \int_{X} \mathcal{H}^{\alpha}(X)^{1 / b}\left(\int_{X} \kappa^{p a}(x, y, z) \mathrm{d} \mathcal{H}^{\alpha}(x)\right)^{1 / a} \mathrm{~d} \mathcal{H}^{\alpha}(y) \mathrm{d} \mathcal{H}^{\alpha}(z) \\
\leq & \mathcal{H}^{\alpha}(X)^{1 / b} \int_{X} \mathcal{H}^{\alpha}(X)^{1 / b}\left[\int_{X}\left(\int_{X} \kappa^{q}(x, y, z) \mathrm{d} \mathcal{H}^{\alpha}(x)\right)^{a \cdot 1 / a} \mathrm{~d} \mathcal{H}^{\alpha}(y)\right]^{1 / a} \mathrm{~d} \mathcal{H}^{\alpha}(z) \\
\leq & \mathcal{H}^{\alpha}(X)^{3 / b}\left(\int_{X}\left[\int_{X} \int_{X} \kappa^{q}(x, y, z) \mathrm{d} \mathcal{H}^{\alpha}(x) \mathrm{d} \mathcal{H}^{\alpha}(y)\right]^{a \cdot 1 / a} \mathrm{~d} \mathcal{H}^{\alpha}(z)\right)^{1 / a} \\
\leq & \mathcal{H}^{\alpha}(X)^{3(1-p / q)}\left(\int_{X} \int_{X} \int_{X} \kappa^{q}(x, y, z) \mathrm{d} \mathcal{H}^{\alpha}(x) \mathrm{d} \mathcal{H}^{\alpha}(y) \mathrm{d} \mathcal{H}^{\alpha}(z)\right)^{p / q} .
\end{aligned}
$$

The inequalities for the other two energies are proven analogously.

Later on we often use the contrapositive of the following lemma to show that a set has infinite curvature energy.

Lemma $2.11\left(\mathcal{F}\left(B_{r}\right) \rightarrow 0\right.$ if $\left.\mathcal{F}(X)<\infty\right)$.

Let $(X, d)$ be a metric space with $\mathcal{H}^{\alpha}(X)<\infty, \alpha, p \in(0, \infty), \mathcal{F} \in\left\{\mathcal{U}_{p}^{\alpha}, \mathcal{I}_{p}^{\alpha}, \mathcal{M}_{p}^{\alpha}\right\}$. If we have finite energy $\mathcal{F}(X)<\infty$ then for all $x \in X$

$$
\lim _{r \downarrow 0} \mathcal{F}\left(B_{r}(x)\right)=0 .
$$

Proof. Let $x_{0} \in X$ and assume that there is a monotonically decreasing sequence $\left(r_{n}\right)_{n \in \mathbb{N}}$, $r_{n}>0$ with $\lim _{n \rightarrow \infty} r_{n}=0$, such that $\mathcal{F}\left(B_{r_{n}}\left(x_{0}\right)\right) \geq c>0$ for all $n \in \mathbb{N}$. We first note that as $B_{r}\left(x_{0}\right) \in \mathcal{C}\left(\mathcal{H}^{\alpha}\right)$ and measures are continuous on monotonically decreasing sets $E_{j}$, if $E_{1}$ has finite measure, [Fal85, Theorem 1.1, (b), p.2] we have

$$
\lim _{n \rightarrow \infty} \mathcal{H}^{\alpha}\left(B_{r_{n}}\left(x_{0}\right)\right)=\mathcal{H}^{\alpha}\left(\lim _{n \rightarrow \infty} B_{r_{n}}\left(x_{0}\right)\right)=\mathcal{H}^{\alpha}\left(\left\{x_{0}\right\}\right)=0 .
$$


Let

$$
f \in\left\{x \mapsto \kappa_{G}^{p}(x), y \mapsto \int_{X} \kappa_{i}^{p}(x, y) \mathrm{d} \mathcal{H}^{\alpha}(x), z \mapsto \int_{X} \int_{X} \kappa^{p}(x, y, z) \mathrm{d} \mathcal{H}^{\alpha}(x) \mathrm{d} \mathcal{H}^{\alpha}(y)\right\}
$$

be the corresponding integrand to $\mathcal{F}$. Then $f$ is measurable, as we have seen in Lemma 2.4 and Lemma 2.7, and

$$
\int_{B_{r_{n}}\left(x_{0}\right)} f \mathrm{~d} \mathcal{H}^{\alpha} \geq \mathcal{F}\left(B_{r_{n}}\left(x_{0}\right)\right) \geq c>0 .
$$

To conclude the proof we employ Lemma 2.12 for the different integrands $f$ and obtain the desired contradiction, namely $\mathcal{F}(X)=\int_{X} f \mathrm{~d} \mathcal{H}^{\alpha}=\infty$.

Lemma 2.12 (Condition for infinite integral).

Let $\mathcal{V}$ be a regular outer measure on $X, f:(X, \mathcal{C}(V)) \rightarrow(\overline{\mathbb{R}}, \mathcal{B}(\overline{\mathbb{R}})), f \geq 0$ measurable and $X_{n+1} \subset X_{n} \subset X, X_{n} \in \mathcal{C}(\mathcal{V})$ for $n \in \mathbb{N}$, such that $\mathcal{V}\left(X_{n}\right) \rightarrow 0$. If

$$
\int_{X_{n}} f d \mathcal{V} \geq c>0 \quad \text { for all } n \in \mathbb{N}
$$

then

$$
\int_{X} f d \mathcal{V}=\infty
$$

Proof. We prove the contrapositive. Let $\int_{X} f \mathrm{~d} \mathcal{V}<\infty$. Then $f\left(1-\chi_{X_{n}}\right)$ are measurable [Fol99, 2.6 Proposition, p.45] and converge pointwise and monotonically increasing to $f$, so that by the monotone convergence theorem, see for example [EG92, 1.3, Theorem 2, p.20] we have

$$
\int_{X} f\left(1-\chi_{X_{n}}\right) \mathrm{d} \mathcal{V}=\int_{X} f \mathrm{~d} \mathcal{V}-\int_{X_{n}} f \mathrm{~d} \mathcal{V} \rightarrow \int_{X} f \mathrm{~d} \mathcal{V}
$$

and hence the proposition.

We also need the following

Lemma 2.13 (Decomposition of triple integral).

Let $\mathcal{V}$ be an outer measure on $X$ and $X_{i} \in \mathcal{C}(\mathcal{V}), i \in \mathbb{N}$ with $\mathcal{V}\left(X_{i} \cap X_{j}\right)=0$ for $i \neq j$ and $X=\bigcup_{i \in \mathbb{N}} X_{i}$. Let $f: X^{3} \rightarrow \overline{\mathbb{R}}, f \geq 0$ be such that for all $x, y, z \in X$ the mappings

$$
x \mapsto f(x, y, z), \quad y \mapsto \int_{X} f(x, y, z) d \mathcal{V}(x) \quad \text { and } \quad z \mapsto \int_{X} \int_{X} f(x, y, z) d \mathcal{V}(x) d \mathcal{V}(y)
$$

$\operatorname{are} \mathcal{C}(\mathcal{V})-\mathcal{B}(\overline{\mathbb{R}})$ measurable. Then

$$
\int_{X} \int_{X} \int_{X} f(x, y, z) d \mathcal{V}(x) d \mathcal{V}(y) d \mathcal{V}(z)=\sum_{i, j, k \in \mathbb{N}} \int_{X_{k}} \int_{X_{j}} \int_{X_{i}} f(x, y, z) d \mathcal{V}(x) d \mathcal{V}(y) d \mathcal{V}(z)
$$

Proof. This is a repeated application of the monotone convergence theorem. If $g: X \rightarrow \overline{\mathbb{R}}$, $g \geq 0$ is $\mathcal{C}(\mathcal{V})-\mathcal{B}(\overline{\mathbb{R}})$ measurable, then so are $g_{n}:=\sum_{i=1}^{n} g \cdot \chi_{X_{i}}$ and $g_{n} \rightarrow g$ monotonically. Hence the monotone convergence theorem gives us

$$
\sum_{i \in \mathbb{N}} \int_{X_{i}} g \mathrm{~d} \mathcal{V}=\lim _{n \rightarrow \infty} \int_{X} g_{n} \mathrm{~d} \mathcal{V}=\int_{X} g \mathrm{~d} \mathcal{V}
$$


Just after the first version of this paper had been written up Martin Meurer, who also did a higher dimensional version of this, and the author could show the following lemma. It offers us the opportunity to include sets with infinite measure in our subsequent theorems.

\section{Lemma 2.14 (Finite energy implies finite measure on all balls).}

Let $\alpha \in[1, \infty), p \in(0, \infty), \mathcal{F} \in\left\{\mathcal{U}_{p}^{\alpha}, \mathcal{I}_{p}^{\alpha}, \mathcal{M}_{p}^{\alpha}\right\}$ and $X \subset \mathbb{R}^{n}$ be a set with $\mathcal{F}(X)<\infty$. Then for all $x \in \mathbb{R}^{n}$ and all $R>0$ we have $\mathcal{H}^{\alpha}\left(X \cap \bar{B}_{R}(x)\right)<\infty$.

Proof. We argue by contradiction and therefore assume that this is not the case.

Step 1 We show that there is an $x_{0} \in \bar{B}_{R}(x)$ with

$$
\mathcal{H}^{\alpha}\left(X \cap B_{r}\left(x_{0}\right)\right)=\infty \quad \text { for all } r>0 \text {. }
$$

According to our assumption there exists $x \in \mathbb{R}^{n}$ and $R>0$, such that $\mathcal{H}^{\alpha}\left(X \cap B_{R}(x)\right)=$ $\infty$. By a covering argument we know that for any $n \in \mathbb{N}$ there is an $x_{n} \in B_{R}(x)$, such that $\mathcal{H}^{\alpha}\left(X \cap B_{1 / n}\left(x_{n}\right)\right)=\infty$. As $\bar{B}_{R}(x)$ is compact, there is a subsequence, such that $x_{n_{k}} \rightarrow x_{0} \in \bar{B}_{R}(x)$. Then $\mathcal{H}^{\alpha}\left(X \cap B_{r}\left(x_{0}\right)\right)=\infty$ for all $r>0$, because

$$
\sup _{y \in B_{1 / n_{k}}\left(x_{n_{k}}\right)} d\left(x_{0}, y\right) \leq d\left(x_{0}, x_{n_{k}}\right)+\frac{1}{n_{k}} \rightarrow 0 .
$$

Step 2 For $\rho>0$ we can find $r=r(\rho), A:=\left[B_{\rho}\left(x_{0}\right) \backslash B_{r}\left(x_{0}\right)\right]$ such that $\mathcal{H}^{\alpha}(X \cap A) \geq 3 \rho$, because $B_{\rho}\left(x_{0}\right) \backslash B_{r}\left(x_{0}\right) \in \mathcal{C}\left(\mathcal{H}_{X}^{\alpha}\right)$ and by Lemma B.4 and the continuity of measures on increasing sets [Fal85, Theorem 1.1, (a), p.2] we have

$$
\begin{aligned}
\mathcal{H}^{\alpha}(X & \left.\cap B_{\rho}\left(x_{0}\right)\right)=\mathcal{H}_{X}^{\alpha}\left(B_{\rho}\left(x_{0}\right)\right)=\mathcal{H}_{X}^{\alpha}\left(B_{\rho}\left(x_{0}\right) \backslash\left\{x_{0}\right\}\right) \\
& =\mathcal{H}_{X}^{\alpha}\left(\bigcup_{n \in \mathbb{N}} B_{\rho}\left(x_{0}\right) \backslash B_{1 / n}\left(x_{0}\right)\right)=\lim _{n \rightarrow \infty} \mathcal{H}_{X}^{\alpha}\left(B_{\rho}\left(x_{0}\right) \backslash B_{1 / n}\left(x_{0}\right)\right)=\infty .
\end{aligned}
$$

Then there exists a direction $s \in \mathbb{S}^{n-1}$ and an $\varepsilon>0$, such that

$$
\mathcal{H}^{\alpha}\left(X \cap A \cap C_{s, \varepsilon}\left(x_{0}\right)\right)>0 \quad \text { and } \quad \mathcal{H}^{\alpha}\left([X \cap A] \backslash C_{s, 2 \varepsilon}\left(x_{0}\right)\right)>0,
$$

because, by a covering and compactness argument similar to that of Step 1, there is a direction $s$, such that for all $\varepsilon>0$ we have $\mathcal{H}^{\alpha}\left(X \cap A \cap C_{s, \varepsilon}\left(x_{0}\right)\right)>0$. If we assume that $\mathcal{H}^{\alpha}\left([X \cap A] \backslash C_{s, 2 \varepsilon}\left(x_{0}\right)\right)=0$ for all $\varepsilon>0$, we obtain a contradiction for $N_{n}:=$ $[X \cap A] \backslash C_{s, 1 / n}\left(x_{0}\right)$ as

$$
\mathcal{H}^{\alpha}([X \cap A] \backslash L)=\mathcal{H}^{\alpha}\left(\bigcup_{n \in \mathbb{N}} N_{n}\right) \leq \sum_{n \in \mathbb{N}} \mathcal{H}^{\alpha}\left(N_{n}\right)=0
$$

by

$$
3 \rho \leq \mathcal{H}^{\alpha}(X \cap A)=\mathcal{H}^{\alpha}([X \cap A] \backslash L)+\mathcal{H}^{\alpha}(X \cap A \cap L)=\mathcal{H}^{\alpha}(X \cap A \cap L) \leq 2 \rho,
$$

where $L=x_{0}+[-\rho, \rho] s$. For the last inequality we needed $\alpha \in[1, \infty)$.

Step 3 Denote $C:=X \cap A \cap C_{s, \varepsilon}\left(x_{0}\right)$ and $C^{\prime}:=[X \cap A] \backslash C_{s, 2 \varepsilon}\left(x_{0}\right)$ the sets from (4). By Lemma C.1 we have $\operatorname{dist}\left(L_{x, y}, x_{0}\right) \geq \sin (\varepsilon) r / 2$ for all $x \in C$ and all $y \in C^{\prime}$, so that for all $z \in B_{\sin (\varepsilon) r / 4}\left(x_{0}\right)$ we have

$$
\operatorname{dist}\left(L_{x, y}, z\right) \geq \operatorname{dist}\left(L_{x, y}, x_{0}\right)-d\left(z, x_{0}\right) \geq \sin (\varepsilon) r / 4
$$


and hence by (3)

$$
\begin{gathered}
\mathcal{M}_{p}^{\alpha}(X) \geq \int_{C} \int_{C^{\prime}} \int_{B_{\sin (\varepsilon) r / 4}\left(x_{0}\right)} \frac{[\sin (\varepsilon) r / 4]^{p}}{r^{2 p}} \mathrm{~d} \mathcal{H}^{\alpha}(z) \mathrm{d} \mathcal{H}^{\alpha}(y) \mathrm{d} \mathcal{H}^{\alpha}(x) \\
\geq \mathcal{H}^{\alpha}(C) \mathcal{H}^{\alpha}\left(C^{\prime}\right) \mathcal{H}^{\alpha}\left(B_{\sin (\varepsilon) r / 4}\left(x_{0}\right)\right) \frac{[\sin (\varepsilon) r / 4]^{p}}{r^{2 p}} \stackrel{(3)}{=} \infty
\end{gathered}
$$

With a similar argument for the other energies we have proven the proposition.

Corollary 2.15 (Finite energy implies that $\mathcal{H}^{\alpha}$ is a Radon measure).

Let $\alpha \in[1, \infty), p \in(0, \infty), \mathcal{F} \in\left\{\mathcal{U}_{p}^{\alpha}, \mathcal{I}_{p}^{\alpha}, \mathcal{M}_{p}^{\alpha}\right\}$ and $X \subset \mathbb{R}^{n}$ be a set with $\mathcal{F}(X)<\infty$. Then $\mathcal{H}_{X}^{\alpha}$ is a Radon measure.

Proof. This is a direct consequence of Lemma 2.14 .

Lemma 2.16 (Consequences of finite energy for $\alpha \in(0,1)$ ). Let $\alpha \in(0,1), p \in(0, \infty), \mathcal{F} \in\left\{\mathcal{U}_{p}^{\alpha}, \mathcal{I}_{p}^{\alpha}, \mathcal{M}_{p}^{\alpha}\right\}$ and $X \subset \mathbb{R}^{n}$ be a set with $\mathcal{F}(X)<\infty$. For all $x_{0} \in X$ we have one of the following propositions

- there is an $\rho>0$, such that $\mathcal{H}^{\alpha}\left(X \cap \bar{B}_{r}\left(x_{0}\right)\right)<\infty$ for all $r \in(0, \rho)$, or

- there is a direction $s \in \mathbb{S}^{n-1}$, such that $\Theta^{\alpha}\left(\mathcal{H}^{\alpha}, X \backslash C_{s, \varepsilon}\left(x_{0}\right), x_{0}\right)=0$ for all $\varepsilon>0$.

Proof. Assume that both alternatives are false, i.e. that

$$
\mathcal{H}^{\alpha}\left(X \cap \bar{B}_{r_{n}}\left(x_{0}\right)\right)=\infty \text { for a sequence } r_{n} \downarrow 0
$$

and for all $s \in \mathbb{S}^{n-1}$ there is $\varepsilon_{s}>0$, such that

$$
\Theta^{* \alpha}\left(\mathcal{H}^{\alpha}, X \backslash C_{s, \varepsilon_{s}}\left(x_{0}\right), x_{0}\right)>0
$$

We show that then $\mathcal{M}_{p}^{\alpha}(X)=\infty$. For the other energies a similar argument can be applied. Denote $A_{r}:=\bar{B}_{\rho}\left(x_{0}\right) \backslash B_{r}\left(x_{0}\right)$ and $L_{s}=x_{0}+\mathbb{R} s$ for a direction $s \in \mathbb{S}^{n-1}$. Due to (5) and an argument similar to that indicated in Step 2 of Lemma 2.14, we can find a direction $s_{0} \in \mathbb{S}^{n-1}$, such that $\mathcal{H}^{\alpha}\left(\left[X \cap A_{r}\right] \cap C_{s_{0}, \varepsilon}\left(x_{0}\right)\right)>0$ for all $\varepsilon>0$ and all $\rho>0$, if $r \in(0, \rho)$ is small enough.

Case 1 We first investigate the case that $\mathcal{H}^{\alpha}\left(X \cap \bar{B}_{r_{n}}\left(x_{0}\right) \cap L_{s_{0}}\right)<\infty$ for $n \geq N$. Now we can argue analogously to Step 2 from Lemma 2.14 to obtain for all $\rho \in\left(0, r_{N}\right)$ a contradiction to $\mathcal{H}^{\alpha}\left(\left[X \cap A_{r}\right] \backslash C_{s_{0}, 2 \varepsilon}\left(x_{0}\right)\right)=0$ for all $\varepsilon>0$ and all $r \in(0, \rho)$ by

$$
\infty \stackrel{(5)}{=} \lim _{r \downarrow 0} \mathcal{H}^{\alpha}\left(X \cap A_{r}\right)=\mathcal{H}^{\alpha}\left(X \cap \bar{B}_{\rho}\left(x_{0}\right) \cap L_{s_{0}}\right) \leq \mathcal{H}^{\alpha}\left(X \cap \bar{B}_{r_{N}}\left(x_{0}\right) \cap L_{s_{0}}\right)<\infty .
$$

Therefore we have shown the analogous result to Step 2 from Lemma 2.14 and can use Step 3 of this lemma to obtain $\mathcal{M}_{p}^{\alpha}(X)=\infty$.

Case 2 It is left to deal with the case that there is a subsequence, such that $\mathcal{H}^{\alpha}(X \cap$ $\left.\bar{B}_{r_{n_{k}}}\left(x_{0}\right) \cap L_{s_{0}}\right)=\infty$ for $k \in \mathbb{N}$. Now we can use $(6)$ to obtain $\mathcal{H}^{\alpha}\left(X \cap B_{r_{n_{k}}}\left(x_{0}\right) \backslash C_{s_{0}, \varepsilon_{s_{0}}}\left(x_{0}\right)\right)>$ 0 . Then we argue again as in Step 3 of Lemma 2.14, using (5), to obtain $\mathcal{M}_{p}^{\alpha}(X)=\infty$. 


\section{$3 \quad$ Hausdorff density and lower estimates of annuli}

In this section we remind the reader of the definition of Hausdorff density, introduce a slightly wider notion for set valued mappings and prove some properties of these densities. More importantly we estimate the Hausdorff measure of annuli from below under the assumption that the densities fulfill certain conditions.

Definition 3.1 (Hausdorff density for set-valued mappings).

Let $(X, d)$ be a metric space, $x \in X, \alpha \in(0, \infty)$ and $A:(0, \rho) \rightarrow \operatorname{Pot}(X)$. Then

$$
\begin{aligned}
\Theta_{*}^{\alpha}\left(\mathcal{H}^{\alpha}, A(r), x\right) & :=\liminf _{r \downarrow 0} \frac{\mathcal{H}^{\alpha}\left(A(r) \cap \bar{B}_{r}(x)\right)}{(2 r)^{\alpha}}, \\
\Theta^{* \alpha}\left(\mathcal{H}^{\alpha}, A(r), x\right) & :=\limsup _{r \downarrow 0} \frac{\mathcal{H}^{\alpha}\left(A(r) \cap \bar{B}_{r}(x)\right)}{(2 r)^{\alpha}}
\end{aligned}
$$

are called the lower and upper $\alpha$-dimensional Hausdorff density of $A$ in $x$. If upper and lower density coincide we call their common value Hausdorff density and denote it by $\Theta^{\alpha}\left(\mathcal{H}^{\alpha}, A(r), x\right)$. Here

$$
\bar{B}_{r}(x):=\{y \in X \mid d(x, y) \leq r\}
$$

is the closed ball of radius $r$ about $x$. If $A(r) \equiv A$ is constant we will usually identify the mapping with the constant and neglect the argument.

Remark 3.2 (Warning: closure of ball $\mathrm{cl}\left(B_{r}(x)\right)$ may not equal closed ball $\bar{B}_{r}(x)$ ). In normed vector spaces the notion of closed balls and the closure of balls coincides. However, in metric spaces this may not be the case, as can be quickly seen by looking at $B_{1}(0)=\{0\}, \operatorname{cl}\left(B_{1}(0)\right)=\{0\}$ and $\bar{B}_{1}(0)=\mathbb{R}$ in $(\mathbb{R}, d)$, where $d$ is the discrete metric.

Lemma 3.3 (Implications of positive lower density).

Let $(X, d)$ be a metric space, $x \in X, A:(0, \varrho) \rightarrow \operatorname{Pot}(X), \alpha \in(0, \infty)$ and $\vartheta_{*}:=$ $\Theta_{*}^{\alpha}\left(\mathcal{H}^{\alpha}, A(r), x\right)>0$. Then for all $\theta \in\left(0,2^{\alpha} \vartheta_{*}\right)$ there is $\rho>0$, such that for all $r \in(0, \rho)$ we have

$$
\theta r^{\alpha} \leq \mathcal{H}^{\alpha}\left(A(r) \cap \bar{B}_{r}(x)\right)
$$

Proof. Fix $\theta \in\left(0,2^{\alpha} \vartheta_{*}\right)$ and assume that the proposition if false. Then for all $\rho>0$ there is $r_{\rho} \in(0, \rho)$, such that

$$
\mathcal{H}^{\alpha}\left(A\left(r_{\rho}\right) \cap \bar{B}_{r_{\rho}}(x)\right)<\theta r_{\rho}^{\alpha} .
$$

Choose $\rho_{n}=n^{-1}$ and obtain a sequence $r_{n^{-1}}$, such that $r_{n^{-1}} \rightarrow 0$ and (7), but this means that $\vartheta_{*}=\Theta_{*}^{\alpha}\left(\mathcal{H}^{\alpha}, A(r), x\right) \leq \theta / 2^{\alpha}$, which contradicts $\theta \in\left(0,2^{\alpha} \vartheta_{*}\right)$.

\section{Lemma 3.4 (Implications of finite upper density).}

Let $(X, d)$ be a metric space, $x \in X, A:(0, \varrho) \rightarrow \operatorname{Pot}(X), \alpha \in(0, \infty)$ and $\vartheta^{*}:=$ $\Theta^{* \alpha}\left(\mathcal{H}^{\alpha}, A(r), x\right)<\infty$. Then for all $\theta \in\left(2^{\alpha} \vartheta^{*}, \infty\right)$, there is $\rho>0$, such that for all $r \in(0, \rho)$ we have

$$
\mathcal{H}^{\alpha}\left(A(r) \cap \bar{B}_{r}(x)\right) \leq \theta r^{\alpha}
$$


Proof. Fix $\theta \in\left(2^{\alpha} \vartheta^{*}, \infty\right)$ and assume that the proposition if false. Then for all $\rho>0$ there is $r_{\rho} \in(0, \rho)$, such that

$$
\theta r_{\rho}^{\alpha}<\mathcal{H}^{\alpha}\left(A\left(r_{\rho}\right) \cap \bar{B}_{r_{\rho}}(x)\right)
$$

Choose $\rho_{n}=n^{-1}$ and obtain a sequence $r_{n^{-1}}$, such that $r_{n^{-1}} \rightarrow 0$ and (8), but this means that $\theta / 2^{\alpha} \leq \Theta^{* \alpha}\left(\mathcal{H}^{\alpha}, A(r), x\right)=\vartheta^{*}$, which contradicts $\theta \in\left(2^{\alpha} \vartheta^{*}, \infty\right)$.

Lemma 3.5 (Simultaneous estimate of annuli).

Let $(X, d)$ be a metric space, $\alpha \in(0, \infty), A, B:(0, \rho) \rightarrow \operatorname{Pot}(X), x \in X$ with

$$
0<\Theta_{*}^{\alpha}\left(\mathcal{H}^{\alpha}, A(r), x\right), \quad 0<\Theta^{* \alpha}\left(\mathcal{H}^{\alpha}, B(r), x\right) \quad \text { and } \quad \Theta^{* \alpha}\left(\mathcal{H}^{\alpha}, X, x\right)<\infty .
$$

Then there exists a $q_{0} \in(0,1)$, a sequence $\left(r_{n}\right)_{n \in \mathbb{N}}, r_{n}>0, \lim _{n \rightarrow \infty} r_{n}=0$ and a constant $c>0$ such that

$$
c r_{n}^{\alpha} \leq \min \left\{\mathcal{H}^{\alpha}\left(A\left(r_{n}\right) \cap\left[\bar{B}_{r_{n}}(x) \backslash B_{q_{0} r_{n}}(x)\right]\right), \mathcal{H}^{\alpha}\left(B\left(r_{n}\right) \cap\left[\bar{B}_{r_{n}}(x) \backslash B_{q_{0} r_{n}}(x)\right]\right)\right\} .
$$

Proof. Step 1 By our hypothesis $\Theta^{* \alpha}\left(\mathcal{H}^{\alpha}, B(r), x\right)=\delta_{0}>0$ and $\Theta^{* \alpha}\left(\mathcal{H}^{\alpha}, X, x\right)=: \theta / 4^{\alpha}<$ $\infty$ there are $r_{n}>0, r_{n} \rightarrow 0$, such that

$$
\delta_{0} r_{n}^{\alpha} \leq \mathcal{H}^{\alpha}\left(B\left(r_{n}\right) \cap \bar{B}_{r_{n}}(x)\right)
$$

and

$$
\mathcal{H}^{\alpha}\left(B\left(r_{n}\right) \cap \bar{B}_{q r_{n}}(x)\right) \leq \mathcal{H}^{\alpha}\left(\bar{B}_{q r_{n}}(x)\right) \leq \theta q^{\alpha} r_{n}^{\alpha} \quad \text { for all } q \in(0,1),
$$

see Lemma 3.4. Together this means that

$$
\begin{aligned}
\mathcal{H}^{\alpha}( & \left.B\left(r_{n}\right) \cap\left[\bar{B}_{r_{n}}(x) \backslash B_{q r_{n}}(x)\right]\right) \\
& \geq \mathcal{H}^{\alpha}\left(B\left(r_{n}\right) \cap \bar{B}_{r_{n}}(x)\right)-\mathcal{H}^{\alpha}\left(B\left(r_{n}\right) \cap B_{q r_{n}}(x)\right) \\
& \geq \mathcal{H}^{\alpha}\left(B\left(r_{n}\right) \cap \bar{B}_{r_{n}}(x)\right)-\mathcal{H}^{\alpha}\left(B\left(r_{n}\right) \cap \bar{B}_{q r_{n}}(x)\right) \\
& \geq\left(\delta_{0}-\theta q^{\alpha}\right) r_{n}^{\alpha} \geq \delta_{0} r_{n}^{\alpha} / 2,
\end{aligned}
$$

if we choose $q^{\alpha} \leq \delta_{0} /(2 \theta)<1$.

Step 2 As $0<\delta_{1}:=\Theta_{*}^{\alpha}\left(\mathcal{H}^{\alpha}, A(r), x\right)$ we know that

$$
\delta_{1} r_{n}^{\alpha} \leq \mathcal{H}^{\alpha}\left(A\left(r_{n}\right) \cap \bar{B}_{r_{n}}(x)\right)
$$

and can use the argument from Step 1 to obtain

$$
\mathcal{H}^{\alpha}\left(A\left(r_{n}\right) \cap\left[\bar{B}_{r_{n}}(x) \backslash B_{q r_{n}}(x)\right]\right) \geq\left(\delta_{1}-\theta q^{\alpha}\right) r_{n}^{\alpha} \geq \delta_{1} r_{n}^{\alpha} / 2
$$

if we choose $q^{\alpha} \leq \delta_{1} /(2 \theta)<1$.

Step 3 Combining the results from the previous steps we obtain the proposition for $q_{0}=\left[\min \left\{\delta_{1}, \delta_{2}\right\} /(2 \theta)\right]^{1 / \alpha} \in(0,1)$ and $c=\min \left\{\delta_{1}, \delta_{2}\right\} / 2$.

Lemma 3.6 (Existence of positive upper density in finite decomposition).

Let $(X, d)$ be a metric space $x \in X, \alpha \in(0, \infty), \Theta^{* \alpha}\left(\mathcal{H}^{\alpha}, X, x\right)>0$ and $X_{i} \subset X$, $i \in\{1, \ldots, N\}$ such that $X=\bigcup_{i=1}^{N} X_{i}$. Then there exists an $n \in\{1, \ldots, N\}$, such that $\Theta^{* \alpha}\left(\mathcal{H}^{\alpha}, X_{n}, x\right)>0$. 
Proof. Assume that this is not the case. Then we obtain a contradiction to $\Theta^{* \alpha}\left(\mathcal{H}^{\alpha}, X, x\right)>$ 0 by

$$
\lim _{n \rightarrow \infty} \frac{\mathcal{H}^{\alpha}\left(X \cap \bar{B}_{r_{n}}(x)\right)}{\left(2 r_{n}\right)^{\alpha}} \leq \lim _{n \rightarrow \infty} \sum_{i=1}^{N} \frac{\mathcal{H}^{\alpha}\left(X_{i} \cap \bar{B}_{r_{n}}(x)\right)}{\left(2 r_{n}\right)^{\alpha}}=0,
$$

for any sequence of radii $\left(r_{n}\right)_{n \in \mathbb{N}}, r_{n}>0, \lim _{n \rightarrow \infty} r_{n}=0$.

Remark 3.7 (Lemma 3.6 is not true for countable decomposition).

If we choose $X=[0,1], X_{0}=\{0\}$ and $X_{n}=\left(2^{-n}, 2^{-n+1}\right]$, we see that $\Theta^{* 1}\left(\mathcal{H}^{1}, X_{n}, 0\right)=0$ for all $n \in \mathbb{N}_{0}$, but $\Theta^{* 1}\left(\mathcal{H}^{1}, X, 0\right)=1 / 2>0$.

Remark 3.8 (In $\mathbb{R}^{n}$ we do not need $x \in X$ ).

Note that for example in case $X \subset \mathbb{R}^{n}$ we do not require $x \in X$ in Lemma 3.3, Lemma 3.4 Lemma 3.5 and Lemma 3.6.

We would like to remind the reader that the angle $\measuredangle\left(s, 0, s^{\prime}\right)$ is a metric, denoted by $d_{\mathbb{S}^{n-1}}\left(s, s^{\prime}\right)$, on the sphere $\mathbb{S}^{n-1}$, so that $\left(\mathbb{S}^{n-1}, d_{\mathbb{S}^{n-1}}\right)$ is a complete metric space.

Lemma 3.9 (Uniform estimate of cones if $\left.\Theta_{*}^{\alpha}\left(\mathcal{H}^{\alpha}, X, x\right)>0\right)$.

Let $X \subset \mathbb{R}^{n}, x \in \mathbb{R}^{n}$ and $\Theta_{*}^{\alpha}\left(\mathcal{H}^{\alpha}, X, x\right)>0$. Then there is a $\rho>0$ and a mapping $s:(0, \rho) \rightarrow \mathbb{S}^{n-1}$, such that for all $\varepsilon>0$ there is $c(\varepsilon)>0$ with

$$
c(\varepsilon) r^{\alpha} \leq \mathcal{H}^{\alpha}\left(X \cap \bar{B}_{r}(x) \cap C_{s(r), \varepsilon}(x)\right) \text { for all } r \in(0, \rho) .
$$

Proof. Step 1 Fix $x \in \mathbb{R}^{n}$. Let $0<\varphi<\psi, s \in \mathbb{S}^{n-1}$ and define

$$
M(s, \alpha, \psi):=\min \left\{|I| \mid C_{s, \psi}(x) \subset \bigcup_{i \in I} C_{s_{i}, \varphi}(x), s_{i} \in \mathbb{S}^{n-1}, d_{\mathbb{S}^{n-1}}\left(s, s_{i}\right)<\psi\right\} .
$$

As $x+\mathbb{S}^{n-1}$ is compact in $\mathbb{R}^{n}$ we can always find a finite subcover of $\bar{C}_{s, \psi}(x)$ in $\left\{C_{s^{\prime}, \varphi}(x) \mid\right.$ $\left.s^{\prime} \in \mathbb{S}^{n-1}, d_{\mathbb{S}^{n-1}}\left(s, s^{\prime}\right)<\psi\right\}$ and consequently $M(s, \varphi, \psi)$ is finite. We can transform the situation for $s$ to that of $\tilde{s}$ by a rotation and hence it is clear that $M(s, \varphi, \psi)=M(\tilde{s}, \varphi, \psi)$ for all $s, \tilde{s} \in \mathbb{S}^{n-1}$. Therefore we write $M(\varphi, \psi):=M(s, \varphi, \psi)$.

Step 2 We define $s_{0}(r):=e_{1}=(1,0, \ldots, 0)$ and $\varepsilon_{0}:=2 \pi 2^{-0}=2 \pi$. From Lemma 3.3 we know that there are $\rho>0$ and $c>0$, such that

$$
\mathcal{H}^{\alpha}\left(X \cap \bar{B}_{r}(X)\right)=\mathcal{H}^{\alpha}\left(X \cap \bar{B}_{r}(X) \cap C_{s_{0}(r), \varepsilon_{0}}(x)\right) \geq c r^{\alpha} \quad \text { for all } r \in(0, \rho) .
$$

Now we set $\varepsilon_{k+1}=2 \pi 2^{-(k+1)}$ and find, with the help of Step 1, a direction $s_{k+1}(r) \in \mathbb{S}^{n-1}$ with $d_{\mathbb{S}^{n-1}}\left(s_{k}(r), s_{k+1}(r)\right)<\varepsilon_{k}$, such that

$$
\begin{aligned}
\mathcal{H}^{\alpha}(X & \left.\cap \bar{B}_{r}(X) \cap C_{s_{k+1}(r), \varepsilon_{k+1}}(x)\right) \geq \frac{\mathcal{H}^{\alpha}\left(X \cap \bar{B}_{r}(X) \cap C_{s_{k}(r), \varepsilon_{k}}(x)\right)}{M\left(\varepsilon_{k+1}, \varepsilon_{k}\right)} \\
& \geq \ldots \geq \frac{c}{\prod_{i=0}^{k} M\left(\varepsilon_{i+1}, \varepsilon_{i}\right)} r^{\alpha} \quad \text { for all } r \in(0, \rho) .
\end{aligned}
$$

Now Lemma A.8 tells us that for all $r \in(0, \rho)$ there are $s(r) \in \mathbb{S}^{n-1}$, such that $s_{k}(r) \rightarrow$ $s(r)$, with

$$
d_{\mathbb{S}^{n-1}}\left(s_{k}(r), s(r)\right) \leq \sum_{i=k}^{\infty} \varepsilon_{i}=\sum_{i=k}^{\infty} 2 \pi 2^{-i}=2 \pi\left[\frac{1}{1-1 / 2}-\frac{1-1 / 2^{-k}}{1-1 / 2}\right]=2 \pi 2^{-(k-1)}=\varepsilon_{k-1} .
$$


Step 3 Let $\varepsilon>0$, then, as $\varepsilon_{k} \rightarrow 0$, there is a $k$, such that $\varepsilon>\varepsilon_{k-1}+\varepsilon_{k}$. Because $d_{\mathbb{S}^{n-1}}\left(s, s^{\prime}\right)+\varphi \leq \psi$ implies $C_{s^{\prime}, \varphi}(x) \subset C_{s, \psi}(x)$ and we already know $d_{\mathbb{S}^{n-1}}\left(s_{k}(r), s(r)\right) \leq$ $\varepsilon_{k-1}$ by Step 2, we have $C_{s_{k}(r), \varepsilon_{k}}(x) \subset C_{s(r), \varepsilon}(x)$ and hence

$$
\begin{aligned}
& \mathcal{H}^{\alpha}\left(X \cap \bar{B}_{r}(x) \cap C_{s(r), \varepsilon}(x)\right) \geq \mathcal{H}^{\alpha}\left(X \cap \bar{B}_{r}(x) \cap C_{s_{k}(r), \varepsilon_{k}}(x)\right) \\
& \geq \frac{c}{\prod_{i=0}^{k-1} M\left(\varepsilon_{i+1}, \varepsilon_{i}\right)} r^{\alpha}=c(\varepsilon) r^{\alpha} \quad \text { for all } r \in(0, \rho) .
\end{aligned}
$$

\section{Approximate tangents, counterexamples}

We now fix our notation regarding the tangency properties we wish to investigate. Also we give some remarks and examples in this context. In this section we finally leave the setting of metric spaces and are from now on only concerned with subsets of $\mathbb{R}^{n}$.

Definition 4.1 (Double cone in direction $s$ with opening angle $\varepsilon$ ).

Let $x \in \mathbb{R}^{n}, s \in \mathbb{S}^{n-1}$ and $\varepsilon>0$. By $C_{s, \varepsilon}(x)$ we denote the open double cone centred at $x$ in direction $s$, i.e.

$$
C_{s, \varepsilon}(x):=\left\{y \in \mathbb{R}^{n} \backslash\{x\} \mid \min \{\measuredangle(y, x, x-s), \measuredangle(y, x, x+s)\}<\varepsilon\right\} .
$$

Definition 4.2 (Weakly $\alpha$-linearly approximable).

We say that a set $X \subset \mathbb{R}^{n}$ is weakly $\alpha$-linearly approximable, $\alpha \in(0, \infty)$ at a point $x \in \mathbb{R}^{n}$, if there is a $\rho>0$ and a mapping $s:(0, \rho) \rightarrow \mathbb{S}^{n-1}$, such that for every $\varepsilon>0$ and every $\delta>0$, there is an $\rho(\varepsilon, \delta) \in(0, \rho)$ with

$$
\mathcal{H}^{\alpha}\left(\left[X \cap \bar{B}_{r}(x)\right] \backslash C_{s(r), \varepsilon}(x)\right) \leq \delta r^{\alpha} \quad \text { for all } r \in(0, \rho(\varepsilon, \delta)) .
$$

Definition 4.3 (Weak and strong approximate $\alpha$-tangents).

Let $X \subset \mathbb{R}^{n}$ be a set and $x \in \mathbb{R}^{n}, \alpha \in(0, \infty)$. We say that $X$ has a (strong) approximate $\alpha$-tangent at $x$, if there is a direction $s \in \mathbb{S}^{n-1}$, such that

$$
\Theta^{\alpha}\left(\mathcal{H}^{\alpha}, X \backslash C_{s, \varepsilon}(x), x\right)=0 \text { for all } \varepsilon>0,
$$

and we say that $X$ has a weak approximate $\alpha$-tangent at $x$, if there is a $\rho>0$ and a mapping $s:(0, \rho) \rightarrow \mathbb{S}^{n-1}$, such that

$$
\Theta^{\alpha}\left(\mathcal{H}^{\alpha}, X \backslash C_{s(r), \varepsilon}(x), x\right)=0 \quad \text { for all } \varepsilon>0 .
$$

We will also sometimes call the direction $s$ and the mapping $s:(0, \rho) \rightarrow \mathbb{S}^{n-1}$ (strong) approximate $\alpha$-tangent and weak approximate $\alpha$-tangent, respectively.

Lemma 4.4 (Weakly $\alpha$-linearly appr. iff weak approximate $\alpha$-tangents). Let $X \subset \mathbb{R}^{n}$ be a set and $x \in \mathbb{R}^{n}, \alpha \in(0, \infty)$. Then the following are equivalent

- $X$ is weakly $\alpha$-linearly approximable at $x$,

- $X$ has weak approximate $\alpha$-tangents at $x$.

Proof. One direction is directly clear from the definitions and the other direction is proven in Lemma 3.4. 


\section{Remark 4.5 (Differences to standard use of terminology).}

We should warn the reader, that our definition of 1-linear approximability and approximate 1-tangents differ from the standard use in literature [Mat95, 15.7 \& 15.10 Definition, p.206 and 15.17 Definition, p.212] in that we refrain from imposing additional density requirements, like $\Theta^{* 1}\left(\mathcal{H}^{1}, X, x\right)>0$ in the case of approximate 1 -tangents. This is simply due to the fact that in the following sections we obtain simpler formulations of our results, because some distinction of cases can be omitted; as we cannot expect a set with finite curvature energy to have positive upper density at any point.

\section{Remark 4.6 (Difference between approximate 1-tangents and tangents).}

What it means for a set to have an approximate 1-tangent at a point is, in some respects, quite different to having an actual tangent at this point. To illustrate this, consider

$$
S:=\{(x, 0) \mid x \in[0,1]\} \cup\left\{\left(x, x^{2}\right) \mid x \in[0,1]\right\} .
$$

As $x \mapsto x^{2}$ is convex there is $r(\varepsilon)$, such that $S \cap B_{r(\varepsilon)}(0) \subset C_{\varepsilon}(0)$ and hence $S$ has an approximate 1-tangent at $(0,0)$, but an arc length parametrisation $\gamma$ of $S$ does not posses a derivative, and hence a tangent, at $\gamma^{-1}((0,0))$.

Example 4.7 (A set with weak appr. but no appr. 1-tangents).

Set $a_{n}:=2^{-n^{n} n^{3}}, A_{n}:=\left[a_{n} / 2, a_{n}\right]$ and

$$
F:=[\bigcup_{n \in \mathbb{N}} \underbrace{A_{2 n} \times\{0\}}_{=: B_{2 n}}] \cup\left[\bigcup_{n \in \mathbb{N}}^{\{0\} \times A_{2 n-1}}\right] .
$$

For $\varepsilon>0$ we have

$$
\begin{aligned}
\mathcal{H}^{1}\left(F \cap C_{e_{1}, \varepsilon}(0) \cap \bar{B}_{a_{2 n}}(0)\right) & \geq \mathcal{H}^{1}\left(\left[a_{2 n} / 2, a_{2 n}\right]\right)=a_{2 n} / 2 \\
\mathcal{H}^{1}\left(F \cap C_{e_{2}, \varepsilon}(0) \cap \bar{B}_{a_{2 n+1}}(0)\right) & \geq \mathcal{H}^{1}\left(\left[a_{2 n+1} / 2, a_{2 n+1}\right]\right)=a_{2 n+1} / 2 .
\end{aligned}
$$

Now (9) tells us that no approximate 1-tangent exists, because for every $s \in \mathbb{S}^{n-1}$ there is $\varepsilon_{s}>0$ and $i_{s} \in\{1,2\}$, such that $C_{e_{i_{s}, \varepsilon_{s}}, \varepsilon_{s}}(0) \cap C_{s, \varepsilon_{s}}(0)=\emptyset$ and hence by (9) there are $r_{n}=r_{n}(s)>0, r_{n} \rightarrow 0$ with

$$
\Theta^{* 1}\left(\mathcal{H}^{1}, F \backslash C_{s, \varepsilon_{s}}(0), 0\right) \geq \lim _{n \rightarrow \infty} \frac{\mathcal{H}^{1}\left(\left[F \cap C_{e_{i_{s}, \varepsilon_{s}}}(0)\right] \cap \bar{B}_{r_{n}}(0)\right)}{2 r_{n}} \geq \frac{1}{4} .
$$

On the other hand we have

$$
\begin{gathered}
\mathcal{H}^{1}\left(\left[F \cap \bar{B}_{r}(0)\right] \backslash C_{e_{1}, \varepsilon}(0)\right) \leq \mathcal{H}^{1}\left(\left[0, a_{2 n+1}\right]\right)=2^{-(2 n+1)^{2 n+1}(2 n+1)^{3}} \\
\leq 2^{-2 n} 2^{-(2 n)^{2 n}(2 n)^{3}-1}=2^{-2 n} \frac{a_{2 n}}{2} \leq 2^{-2 n} r
\end{gathered}
$$

for all $r \in\left[a_{2 n} / 2, a_{2 n-1} / 2\right]$ and

$$
\begin{gathered}
\mathcal{H}^{1}\left(\left[F \cap \bar{B}_{r}(0)\right] \backslash C_{e_{2}, \varepsilon}(0)\right) \leq \mathcal{H}^{1}\left(\left[0, a_{2(n+1)}\right]\right)=2^{-(2[n+1])^{2[n+1]}(2[n+1])^{3}} \\
\leq 2^{-(2 n+1)} 2^{-(2 n+1)^{2 n+1}(2 n+1)^{3}-1}=2^{-(2 n+1)} \frac{a_{2 n+1}}{2} \leq 2^{-(2 n+1)} r
\end{gathered}
$$

for all $r \in\left(a_{2 n+1} / 2, a_{2 n} / 2\right)$. We therefore have verified the definition of $F$ having a weak approximate 1-tangent for

$$
s:(0,1 / 2) \rightarrow \mathbb{S}^{1}, r \mapsto \begin{cases}e_{1}, & r \in \bigcup_{n \in \mathbb{N}}\left[a_{2 n} / 2, a_{2 n-1} / 2\right], \\ e_{2}, & r \in \bigcup_{n \in \mathbb{N}}\left(a_{2 n+1} / 2, a_{2 n} / 2\right) .\end{cases}
$$


One might be tempted to think that a continuum with approximate 1-tangents is a topological 1-manifold, i.e. a closed curve or an arc. That these two concepts are not related can be seen by the following remark. If the reader is not familiar with the notion of ramification order we refer him to [BM70, Definition 13.5, p.442 f.].

Remark 4.8 (Relationship between appr. 1-tangents and ramification points). If a set $M$ has an approximate 1-tangent at $x \in M$ then $x$ can still be a ramification point. Let $S$ be the set from Remark 4.6. Then $S \cup([-1,0] \times\{0\})$ has an approximate 1-tangent at 0 and 0 is a point of order 3 . On the other hand a point of order less than 2 does not imply that the set has an approximate 1-tangent at this point. This can be sen as follows: let $M:=([0,1] \times\{0\}) \cup(\{0\} \times[0,1])$. Then 0 is a point of order 2 in $M$, but $M$ does not even possess a weak approximate 1 -tangent at 0 .

\section{Lemma 4.9 (Density estimates for set with no approximate tangent).}

Let $X \subset \mathbb{R}^{n}, x \in \mathbb{R}^{n}, \alpha \in(0, \infty)$ and $\Theta^{* \alpha}\left(\mathcal{H}^{\alpha}, X, x\right)>0$. If $X$ has no approximate $\alpha$-tangent at $x$, then there is $s \in \mathbb{S}^{n-1}$ and $\varepsilon_{0}>0$, such that

$$
0<\Theta^{* \alpha}\left(\mathcal{H}^{\alpha}, X \cap C_{s, \varepsilon_{0} / 2}(x), x\right) \quad \text { and } \quad 0<\Theta^{* \alpha}\left(\mathcal{H}^{\alpha}, X \backslash C_{s, \varepsilon_{0}}(x), x\right) .
$$

Proof. Assuming that there exists no approximate $\alpha$-tangent at $x \in X$ we know that for all directions $s \in \mathbb{S}^{n-1}$ there is an $\varepsilon_{s}>0$, such that $\Theta^{* \alpha}\left(\mathcal{H}^{\alpha}, X \backslash C_{s, \varepsilon_{s}}(x), x\right)>0$. As $\mathbb{S}^{n-1}$ is compact and $\left\{C_{s, \varepsilon_{s} / 2}(x)\right\}_{s \in \mathbb{S}^{n-1}}$ is an open cover of $x+\mathbb{S}^{n-1}$ there exists a finite subcover $\left\{C_{s_{i}, \varepsilon_{s_{i}} / 2}(x)\right\}_{i=1}^{N}$. Clearly this subcover also covers the whole $\mathbb{R}^{n} \backslash\{x\}$. As $0<\Theta^{* \alpha}\left(\mathcal{H}^{\alpha}, X, x\right)=\Theta^{* \alpha}\left(\mathcal{H}^{\alpha}, X \backslash\{x\}, x\right)$ we know, by Lemma 3.6, note Remark 3.8, that for some $j \in\{1, \ldots, N\}$ we have $\Theta^{* \alpha}\left(\mathcal{H}^{\alpha}, X \cap C_{s_{j}, \varepsilon_{j} / 2}(x), x\right)>0$.

Lemma 4.10 (Density estimates for set with no weak approximate tangent). Let $X \subset \mathbb{R}^{n}, x \in \mathbb{R}^{n}, \alpha \in(0, \infty)$ and $\Theta_{*}^{\alpha}\left(\mathcal{H}^{\alpha}, X, x\right)>0$. If $X$ has no weak approximate $\alpha$-tangent at $x$, then there is a mapping $s:(0, \rho) \rightarrow \mathbb{S}^{n-1}, \rho>0$ and $\varepsilon_{0}>0$, such that

$$
0<\Theta_{*}^{\alpha}\left(\mathcal{H}^{\alpha}, X \cap C_{s(r), \varepsilon_{0} / 2}(x), x\right) \quad \text { and } \quad 0<\Theta^{* \alpha}\left(\mathcal{H}^{\alpha}, X \backslash C_{s(r), \varepsilon_{0}}(x), x\right) .
$$

Proof. If $X$ has no weak approximate $\alpha$-tangent at $x \in \mathbb{R}^{n}$ it is not weakly $\alpha$-linearly approximable in $x$, by Lemma 4.4 , so that for all $\rho>0$ and all mappings $s:(0, \rho) \rightarrow \mathbb{S}^{n-1}$ there is an $\varepsilon_{0}>0$ and a $\delta_{0}>0$, such that for all $\rho^{\prime} \in(0, \rho)$ there is $r \in\left(0, \rho^{\prime}\right)$ with

$$
\delta_{0} r^{\alpha}<\mathcal{H}^{\alpha}\left(\left[X \cap \bar{B}_{r}(x)\right] \backslash C_{s(r), \varepsilon_{0}}(x)\right) .
$$

By choosing $\rho^{\prime}=\rho(2 k)^{-1}$ we obtain a sequence $\left(r_{k}\right)_{k \in \mathbb{N}}, r_{k}>0, r_{k} \rightarrow 0$, with

$$
\delta_{0} r_{k}^{\alpha}<\mathcal{H}^{\alpha}\left(\left[X \cap \bar{B}_{r_{k}}(x)\right] \backslash C_{s\left(r_{k}\right), \varepsilon_{0}}(x)\right) \text { for all } k \in \mathbb{N} \text {. }
$$

Now fix $\rho$ and $s:(0, \rho) \rightarrow \mathbb{S}^{n-1}$ to be those we obtain from Lemma 3.9. Then

$$
0<\Theta^{* \alpha}\left(\mathcal{H}^{\alpha}, X \backslash C_{s(r), \varepsilon_{0}}(x), x\right) .
$$

by (10) and Lemma 3.9 gives us

$$
0<c\left(\varepsilon_{0} / 2\right) / 2 \leq \Theta_{*}^{\alpha}\left(\mathcal{H}^{\alpha}, X \cap C_{s(r), \varepsilon_{0} / 2}(x), x\right) .
$$


We shall now give a construction that guarantees that a set has no weak approximate $\alpha$-tangent.

Lemma 4.11 (Construction of sets with no weak appr. tangent).

Let $X \subset \mathbb{R}^{n}, \alpha \in(0, \infty)$ such that $X$ has an approximate $\alpha$-tangent in direction $s \in \mathbb{S}^{n-1}$ at $x \in \mathbb{R}^{n}$ and $\Theta^{* \alpha}\left(\mathcal{H}^{\alpha}, X, x\right)>0$. Let $A \in \mathrm{SO}(n) \backslash\{\mathrm{id}\}, \varphi_{A}(y)=A(y-x)+x$ such that the axis of rotation does not coincide with $\mathbb{R} s$. Then $X \cup \varphi_{A}(X)$ has no weak approximate $\alpha$-tangent at $x$.

Proof. Clearly $\varphi_{A}(X)$ has an approximate $\alpha$-tangent in direction $\varphi_{A}(s)$, so that $X \cup \varphi_{A}(X)$ cannot have a weak approximate $\alpha$-tangent.

\section{$5 \quad$ Finite $1 / \Delta, \mathcal{U}_{p}^{\alpha}$ implies app. $\alpha$-tangents for $p \in[\alpha, \infty)$}

We now show that for $p \in[\alpha, \infty)$ a set with finite $\mathcal{U}_{p}^{\alpha}$ is guaranteed to have approximate $\alpha$-tangents at all points. This directly implies similar results for the inverse thickness $1 / \Delta$. Later on we give a counterexample to the analogous result for $\alpha=1$ and $p \in(0,1)$.

Lemma 5.1 (Finite $\mathcal{U}_{p}^{\alpha}$ guarantees approximate $\alpha$-tangents).

Let $X \subset \mathbb{R}^{n}, x \in \mathbb{R}^{n}, \alpha \in(0, \infty), p \in[\alpha, \infty)$ and $\mathcal{U}_{p}^{\alpha}(X)<\infty$. Then $X$ has an approximate $\alpha$-tangent at $x$.

Proof. Assume that $\Theta^{* \alpha}\left(\mathcal{H}^{\alpha}, X, x\right)>0$ - which we might without loss of generality, because else the proposition is clear - and that $X$ has no approximate $\alpha$-tangent at $x$. As $x$ has to be an accumulation point of $X$ we can, by means of Lemma B.3, assume that without loss of generality $x \in X$. By Lemma 2.14 and Lemma 2.16 we can also assume that $\mathcal{H}^{\alpha}\left(X \cap B_{r}(x)\right)<\infty$ for all small radii. Now we use Lemma 4.9 and set $A:=X \cap C_{s, \varepsilon / 2}(x), B:=X \backslash C_{s, \varepsilon}(x)$ and choose a sequence of radii $r_{n} \downarrow 0$, such that $\mathcal{H}^{\alpha}\left(A \cap \bar{B}_{r_{n}}(x)\right) / r_{n}^{\alpha} \geq c>0$. Then $\measuredangle(a, x, b) \in[\varepsilon / 2, \pi-\varepsilon / 2]$ for all $a \in A$ and all $b \in B$. Clearly $x$ is an accumulation point of of $B$, so that for each $n \in \mathbb{N}$ there exists $b_{n} \in B \cap \bar{B}_{r_{n}}(x)$. Using Lemma C.1 we obtain for all $a \in A \cap \bar{B}_{r_{n}}(x) \backslash\{x\}$

$$
\begin{aligned}
\kappa_{G}(a) & \geq \frac{1}{r\left(a, b_{n}, x\right)}=\frac{2 \operatorname{dist}\left(L_{\left.a, b_{n}, x\right)}\right.}{\|a-x\|\left\|b_{n}-x\right\|} \geq \frac{\sin (\varepsilon / 2) \min \left\{\|a-x\|,\left\|b_{n}-x\right\|\right\}}{\|a-x\|\left\|b_{n}-x\right\|} \\
& =\frac{\sin (\varepsilon / 2)}{\max \left\{\|a-x\|,\left\|b_{n}-x\right\|\right\}} \geq \frac{\sin (\varepsilon / 2)}{r_{n}} .
\end{aligned}
$$

We have

$$
\begin{gathered}
\mathcal{U}_{p}^{\alpha}\left(B_{2 r_{n}}(x) \cap X\right) \geq \int_{A \cap \bar{B}_{r_{n}}(x) \backslash\{x\}} \kappa_{G}^{p}(t) \mathrm{d} \mathcal{H}^{\alpha}(t) \geq \mathcal{H}^{\alpha}\left(A \cap \bar{B}_{r_{n}}(x)\right)\left(\frac{\sin (\varepsilon / 2)}{r_{n}}\right)^{p} \\
\geq c r_{n}^{\alpha}\left(\frac{\sin (\varepsilon / 2)}{r_{n}}\right)^{p} \geq c^{\prime}>0
\end{gathered}
$$

for all $n \in \mathbb{N}$. Hence Lemma 2.11 tells us that $\mathcal{U}_{p}^{\alpha}(X)=\infty$, note that for this we needed $\mathcal{H}^{\alpha}\left(B_{2 r_{n}}(x) \cap X\right)<\infty$. This is absurd as $\mathcal{U}_{p}^{\alpha}(X)<\infty$.

Corollary 5.2 (Sets with finite $\mathcal{U}_{p}^{1}$ are rectifiable).

Let $X \subset \mathbb{R}^{n}$ be an $\mathcal{H}^{1}$-measurable set and $p \in[1, \infty)$. If $\mathcal{U}_{p}^{1}(X)<\infty$ then $X$ is 1 rectifiable. 
Proof. For all $n \in \mathbb{N}$ Lemma 2.14 tells us that $X \cap B_{n}(0)$ has finite measure, so that by Lemma 5.1 together with the equivalent characterisation of rectifiablity in terms of approximate 1-tangents, see for example [Mat95, 15.19 Theorem, p. 212], we know that all $X \cap B_{n}(0)$ are rectifiable. By taking all the rectifiable curves that cover the $X \cap B_{n}(0)$, which are still countably many, we have covered $X$ with countably many curves, so that $X$ is rectifiable.

Corollary 5.3 (Sets with positive thickness are rectifiable).

Let $X \subset \mathbb{R}^{n}$ be an $\mathcal{H}^{1}$-measurable set with $\mathcal{H}^{1}(X)<\infty$ and $1 / \Delta[X]<\infty$. Then $X$ is 1-rectifiable and has an approximate 1 -tangent at each point $x \in \mathbb{R}^{n}$.

Proof. Because $\mathcal{U}_{p}^{1}(X) \leq\left[\mathcal{H}^{1}(X)\right]^{p} / \Delta[X]$, see Lemma 2.9, this a an immediate consequence of Lemma 5.2. The result for the approximate 1-tangents remains true when $X$ is not measurable, but meets the other hypotheses.

\section{$5.1 \quad$ Finite $\mathcal{U}_{p}^{1}$ does not imply (weak) approx. tangents for $p \in(0,1)$}

For further reference we define

Definition 5.4 (The set $E$ ).

We set $E:=([-1,1] \times\{0\}) \cup(\{0\} \times[0,1]) \subset \mathbb{R}^{2}$ as well as $E_{1}:=[-1,0] \times\{0\}, E_{2}:=$ $\{0\} \times[0,1]$ and $E_{3}:=[0,1] \times\{0\}$.

Clearly $E$ does not have a weak approximate 1-tangent at $(0,0)$. To show that our results are sharp, we need to compute the appropriate energy of $E$ in each section. We therefore start with

Proposition 5.5 (The set $E$ has finite $\mathcal{U}_{p}^{1}$ for $p \in(0,1)$ ).

For $p \in(0,1)$ we have

$$
\mathcal{U}_{p}^{1}(E) \leq \frac{6}{1-p}
$$

Proof. For all $x \in E \backslash\{0\}$ and $y, z \in B_{\|x\|}(x) \cap E, y \neq z$ we have $\kappa(x, y, z)=0$, so that for $\kappa(x, y, z)>0$ we need $\|x-y\| \geq\|x\|$ or $\|x-z\| \geq\|x\|$, which both result in $r(x, y, z) \geq\|x\| / 2$ and consequently

$$
\sup _{\substack{y, z \in E \backslash\{x\} \\ y \neq z}} \kappa(x, y, z) \leq \frac{2}{\|x\|},
$$

so that for $p \in(0,1)$

$$
\begin{aligned}
\mathcal{U}_{p}^{1}(E) & =\int_{E \backslash\{0\}} \underbrace{\left(\sup _{\substack{y, z \in E \backslash\{x\} \\
y \neq z}} \kappa(x, y, z)\right)^{p} \mathrm{~d} \mathcal{H}^{1}(x)}_{\leq 2 /\|x\|} \\
& \leq 3 \int_{E_{2}} \frac{2}{\|x\|^{p}} \mathrm{~d} \mathcal{H}^{1}(x)=6 \int_{0}^{1} \frac{1}{s^{p}} \mathrm{~d} \mathcal{L}^{1}(s)=\frac{6}{1-p}<\infty .
\end{aligned}
$$




\section{$6 \quad$ Finite $\mathcal{I}_{p}^{\alpha}$ implies weak app. tangents for $p \in[2 \alpha, \infty)$}

The purpose of this section is to show that for $p \in[2 \alpha, \infty)$ a set with finite $\mathcal{I}_{p}^{\alpha}$ has a weak approximate $\alpha$-tangent at all points where the lower density is positive. We also show that this is not true if $\alpha=1$ and $p \in(0,2)$.

Lemma 6.1 (Necessary conditions for finite $\mathcal{I}_{p}^{\alpha}$ ).

Let $X \subset \mathbb{R}^{n}, z_{0} \in \mathbb{R}^{n}, \alpha \in(0, \infty), \mathcal{H}^{\alpha}(X)<\infty$. Let $\varepsilon>0, c>0$ and two sequences of sets $A_{n}, B_{n} \subset X$ as well as a sequence $\left(r_{n}\right)_{n \in \mathbb{N}}, r_{n}>0, r_{n} \rightarrow 0$ be given, with the following properties:

- for all $n \in \mathbb{N}$ and all $x \in A_{n} \backslash\left\{z_{0}\right\}$ and $y \in B_{n} \backslash\left\{z_{0}\right\}$ we have $\pi-\varepsilon \geq \measuredangle\left(x, z_{0}, y\right) \geq \varepsilon$,

- for all $n \in \mathbb{N}$ we have

$$
c r_{n}^{\alpha} \leq \min \left\{\mathcal{H}^{\alpha}\left(A_{n} \cap \bar{B}_{r_{n}}\left(z_{0}\right)\right), \mathcal{H}^{\alpha}\left(B_{n} \cap \bar{B}_{r_{n}}\left(z_{0}\right)\right)\right\} .
$$

Then $\mathcal{I}_{p}^{\alpha}(X)=\infty$ for all $p \geq 2 \alpha$.

Proof. Let $p \geq 2 \alpha$ and suppose for contradiction that $\mathcal{I}_{p}^{\alpha}(X)<\infty$. As $z_{0}$ has to be an accumulation point of $X$ we can, by means of Lemma B.3, assume that without loss of generality $z_{0} \in X$. If we set

$$
\tilde{A}_{n}:=A_{n} \cap \bar{B}_{r_{n}}\left(z_{0}\right) \quad \text { and } \quad \tilde{B}_{n}:=B_{n} \cap \bar{B}_{r_{n}}\left(z_{0}\right)
$$

Lemma C.1 gives us

$$
\begin{gathered}
\kappa_{i}(x, y) \geq \kappa\left(x, y, z_{0}\right)=\frac{2 \operatorname{dist}\left(L_{x, y}, z_{0}\right)}{\left\|x-z_{0}\right\|\left\|y-z_{0}\right\|} \geq \frac{\sin (\varepsilon / 2) \min \left\{\left\|x-z_{0}\right\|,\left\|y-z_{0}\right\|\right\}}{\left\|x-z_{0}\right\|\left\|y-z_{0}\right\|} \\
=\frac{\sin (\varepsilon / 2)}{\max \left\{\left\|x-z_{0}\right\|,\left\|y-z_{0}\right\|\right\}} \geq \frac{\sin (\varepsilon / 2)}{r_{n}},
\end{gathered}
$$

for all $x \in \tilde{A}_{n} \backslash\left\{z_{0}\right\}$ and $y \in \tilde{B}_{n} \backslash\left\{z_{0}\right\}$. Now we have

$$
\begin{aligned}
\mathcal{I}_{p}^{\alpha}(X & \left.\cap B_{2 r_{n}}\left(z_{0}\right)\right) \geq \mathcal{I}_{p}^{\alpha}\left(X \cap \bar{B}_{r_{n}}\left(z_{0}\right)\right)=\int_{X \cap \bar{B}_{r_{n}}\left(z_{0}\right)} \int_{X \cap \bar{B}_{r_{n}}\left(z_{0}\right)} \kappa_{i}^{p}(x, y) \mathrm{d} \mathcal{H}^{\alpha}(x) \mathrm{d} \mathcal{H}^{\alpha}(y) \\
& \geq \int_{\tilde{B}_{n}} \int_{\tilde{A}_{n}} \kappa_{i}^{p}(x, y) \mathrm{d} \mathcal{H}^{\alpha}(x) \mathrm{d} \mathcal{H}^{\alpha}(y) \geq \mathcal{H}^{\alpha}\left(\tilde{B}_{n}\right) \mathcal{H}^{\alpha}\left(\tilde{A}_{n}\right)\left(\frac{\sin (\varepsilon / 2)}{r_{n}}\right)^{p} \\
& \geq c^{2} \sin ^{p}(\varepsilon / 2) r_{n}^{2 \alpha-p} \geq c^{\prime}>0
\end{aligned}
$$

for $p \geq 2 \alpha$ and all $n \in \mathbb{N}$. Hence Lemma 2.11 tells us that $\mathcal{I}_{p}^{\alpha}(X)=\infty$, note that for this we needed $\mathcal{H}^{\alpha}\left(B_{2 r_{n}}(x) \cap X\right)<\infty$. This is absurd as we assumed $\mathcal{I}_{p}^{\alpha}(X)<\infty$.

Proposition 6.2 (Finite $\mathcal{I}_{p}^{\alpha}, p \geq 2 \alpha$ implies weak app. $\alpha$-tangents).

Let $X \subset \mathbb{R}^{n}$ be a set, $\alpha \in(0, \infty)$ and $x \in \mathbb{R}^{n}$ with $0<\Theta_{*}^{\alpha}\left(\mathcal{H}^{\alpha}, X, x\right)$. If $p \in[2 \alpha, \infty)$ and $\mathcal{I}_{p}^{\alpha}(X)<\infty$ then $X$ has a weak approximate $\alpha$-tangent at $x$.

Proof. Assume that this is not the case. By Lemma 2.14 and Lemma 2.16 we can without loss of generality assume that $\mathcal{H}^{\alpha}\left(X \cap \bar{B}_{r}(x)\right)<\infty$ for all small radii. Then by Lemma 4.10 there is a mapping $s:(0, \rho) \rightarrow \mathbb{S}^{n-1}, \rho>0$ and $\varepsilon_{0}>0$, such that

$$
0<\Theta_{*}^{\alpha}\left(\mathcal{H}^{\alpha}, X \cap C_{s(r), \varepsilon_{0} / 2}(x), x\right)
$$


and

$$
0<\Theta^{* \alpha}\left(\mathcal{H}^{1}, X \backslash C_{s(r), \varepsilon_{0}}(x), x\right) .
$$

This means that there is a constant $c>0$ and a sequence $\left(r_{n}\right)_{n \in \mathbb{N}}, r_{n}>0, r_{n} \rightarrow 0$, such that

$$
c r_{n}^{\alpha} \leq \min \left\{\mathcal{H}^{\alpha}\left(\left[X \cap C_{s\left(r_{n}\right), \varepsilon_{0} / 2}(x)\right] \cap \bar{B}_{r_{n}}(x)\right), \mathcal{H}^{\alpha}\left(\left[X \backslash C_{s\left(r_{n}\right), \varepsilon_{0}}(x)\right] \cap \bar{B}_{r_{n}}(x)\right)\right\}
$$

and hence the hypotheses of Lemma 6.1 hold for

$$
A_{n}:=\left[X \cap \bar{B}_{r}(x)\right] \cap C_{s\left(r_{n}\right), \varepsilon_{0} / 2}(x) \quad \text { and } \quad B_{n}:=\left[X \cap \bar{B}_{r}(x)\right] \backslash C_{s\left(r_{n}\right), \varepsilon_{0}}(x)
$$

for $r$ small enough, i.e. the role $X$ in Lemma 6.1 is played by $X \cap \bar{B}_{r}(x)$, and $\varepsilon:=\varepsilon_{0} / 2$, so that we have proven the proposition.

\subsection{Finite $\mathcal{I}_{p}^{1}$ does not imply (weak) approx. tangents for $p \in(0,2)$}

Proposition 6.3 (The set $E$ has finite $\mathcal{I}_{p}^{1}$ for $p \in(1,2)$ ).

Let $E$ be the set from Definition 5.4. For $p \in(1,2)$ we have

$$
\mathcal{I}_{p}^{1}(E) \leq \frac{9 \cdot 2^{3 p / 2+1}\left(2^{1-p}-1\right)}{(1-p)(2-p)}
$$

Proof. Let $x, y \in E \backslash\{0\}, x \neq y$. We are interested in the maximal value of $\kappa(x, y, z)$ for $z \in E \backslash\{x, y\}$. As $\kappa$ is invariant under isometries we can restrict ourselves to the cases $x, y \in E_{1}$ and $x \in E_{1}, y \in E_{3}$ and $x \in E_{1}, y \in E_{2}$. In each of these cases we want to estimate $\kappa(x, y, z)$ independently of $z$. We denote the non-zero components of $x, y, z$ by $\xi, \eta, \zeta$ respectively.

Case 1 If $x, y \in E_{1}, x y \neq 0$ we clearly can assume $z \in E_{2} \backslash\{0\}$ and hence

$$
\kappa(x, y, z)=\frac{2 \zeta}{\sqrt{\xi^{2}+\zeta^{2}} \sqrt{\eta^{2}+\zeta^{2}}}=\frac{2}{\sqrt{\zeta^{2}+\xi^{2}+\eta^{2}+\xi^{2} \eta^{2} / \zeta^{2}}} .
$$

By taking first and second derivatives of $f(u)=\alpha u+\beta / u, \alpha, \beta>0$, we easily see that $\min _{u>0} f(u)=f(\sqrt{\beta / \alpha})$, so that for all $\zeta>0$ we have

$$
\zeta^{2}+\frac{\xi^{2} \eta^{2}}{\zeta^{2}} \geq \xi \eta+\frac{\xi^{2} \eta^{2}}{\xi \eta}=2 \xi \eta
$$

and therefore

$$
\kappa(x, y, z) \leq \frac{2}{\sqrt{\xi^{2}+\eta^{2}+2 \xi \eta}}=\frac{2}{|\xi|+|\eta|} .
$$

Case 2 If $x \in E_{1}, y \in E_{3}, x y \neq 0$ we do need $z \in E_{2}$ in order to have $\kappa(x, y, z)>0$, but then $\kappa(x, y, z)=\kappa(x,-y, z)$, so that we can without loss of generality assume that $y \in E_{1}$. This was already done in Case 1 .

Case 3 If $x \in E_{1}, y \in E_{2}, x y \neq 0$ we note that we have $\kappa(x, y, z)=\kappa(x, y,-z)$ for $z \in E_{3}$, so that we may assume $z \in E_{1}$ without loss of generality. Then

$$
\kappa(x, y, z)=\frac{2 \eta}{\sqrt{\xi^{2}+\eta^{2}} \sqrt{\zeta^{2}+\eta^{2}}} \leq \frac{2 \eta}{\sqrt{\xi^{2}+\eta^{2}} \sqrt{\eta^{2}}}=\frac{2}{\sqrt{\xi^{2}+\eta^{2}}} \leq \frac{2 \sqrt{2}}{|\xi|+\eta} .
$$


In all cases we have

$$
\kappa(x, y, z) \leq \frac{2 \sqrt{2}}{|\xi|+|\eta|} \quad \text { for all } z \in E \backslash\{x, y\}
$$

which for $p \in(1,2)$ gives us

$$
\begin{aligned}
\mathcal{I}_{1}^{2}(E) & \leq 9 \cdot 2^{3 p / 2} \int_{0}^{1} \int_{0}^{1}\left(\frac{1}{s+t}\right)^{p} \mathrm{~d} \mathcal{L}^{1}(s) \mathrm{d} \mathcal{L}^{1}(t) \\
& =\frac{9 \cdot 2^{3 p / 2}}{1-p} \int_{0}^{1}\left[(1+t)^{1-p}-t^{1-p}\right] \mathrm{d} \mathcal{L}^{1}(t)=\frac{9 \cdot 2^{3 p / 2}}{(1-p)(2-p)}\left[\left[(1+t)^{2-p}-t^{2-p}\right]\right]_{0}^{1} \\
& =\frac{9 \cdot 2^{3 p / 2}}{(1-p)(2-p)}\left[\left[2^{2-p}-1\right]-[1-0]\right]=\frac{9 \cdot 2^{(3 p / 2)+1}\left(2^{1-p}-1\right)}{(1-p)(2-p)} .
\end{aligned}
$$

Corollary 6.4 (The set $E$ has finite $\mathcal{I}_{p}^{1}$ for $p \in(0,2)$ ).

For $p \in(0,2)$ we have $\mathcal{I}_{p}^{1}(E)<\infty$.

Proof. This is a consequence of Lemma 6.3 together with $\mathcal{H}^{1}(E)=3$ and Lemma 2.10.

\section{$7 \quad$ Finite $\mathcal{M}_{p}^{\alpha}$ implies weak app. tangents for $p \in[3 \alpha, \infty)$}

In this section we show that for $p \in[3 \alpha, \infty)$ a set with finite upper density and finite $\mathcal{M}_{p}^{\alpha}$ has a weak approximate $\alpha$-tangent at all points where the lower density is positive. After this we demonstrate that this is not true for $\alpha=1$ and $p \in(0,3)$.

\section{Lemma 7.1 (Necessary conditions for finite Menger curvature).}

Let $X \subset \mathbb{R}^{n}, z_{0} \in \mathbb{R}^{n}, \alpha \in(0, \infty), \mathcal{H}^{\alpha}(X)<\infty, \Theta_{*}^{\alpha}\left(\mathcal{H}^{\alpha}, X, z_{0}\right)>0$. Let $\varepsilon>0, c>0$, $q_{0} \in(0,1)$ and two sequences of sets $A_{n}, B_{n} \subset X$ as well as a sequence $\left(r_{n}\right)_{n \in \mathbb{N}}, r_{n}>0$, $r_{n} \rightarrow 0$ be given, with the following properties:

- for all $n \in \mathbb{N}$ and all $x \in A_{n} \backslash\left\{z_{0}\right\}$ and $y \in B_{n} \backslash\left\{z_{0}\right\}$ we have $\pi-\varepsilon \geq \measuredangle\left(x, z_{0}, y\right) \geq \varepsilon$,

- for all $n \in \mathbb{N}$ we have

$$
c r_{n}^{\alpha} \leq \min \left\{\mathcal{H}^{\alpha}\left(A_{n} \cap\left[\bar{B}_{r_{n}}\left(z_{0}\right) \backslash B_{q_{0} r_{n}}\left(z_{0}\right)\right]\right), \mathcal{H}^{\alpha}\left(B_{n} \cap\left[\bar{B}_{r_{n}}\left(z_{0}\right) \backslash B_{q_{0} r_{n}}\left(z_{0}\right)\right]\right)\right\} .
$$

Then $\mathcal{M}_{p}^{\alpha}(X)=\infty$ for all $p \geq 3 \alpha$.

Proof. Let $p \geq 3 \alpha$ and suppose for contradiction that $\mathcal{M}_{p}^{\alpha}(X)<\infty$. We set

$$
\tilde{A}_{n}:=A_{n} \cap\left[\bar{B}_{r_{n}}\left(z_{0}\right) \backslash B_{q_{0} r_{n}}\left(z_{0}\right)\right] \quad \text { and } \quad \tilde{B}_{n}:=B_{n} \cap\left[\bar{B}_{r_{n}}\left(z_{0}\right) \backslash B_{q_{0} r_{n}}\left(z_{0}\right)\right] \text {. }
$$

Considering Lemma C.1 we know that for all $x \in \tilde{A}_{n} \backslash\left\{z_{0}\right\}$ and $y \in \tilde{B}_{n} \backslash\left\{z_{0}\right\}$ we have $\operatorname{dist}\left(L_{x, y}, z_{0}\right) \geq \sin (\varepsilon) q_{0} r_{n} / 2$ and therefore for all $z \in B_{\sin (\varepsilon) q_{0} r_{n} / 4}\left(z_{0}\right)$

$$
\operatorname{dist}\left(L_{x, y}, z\right) \geq \operatorname{dist}\left(L_{x, y}, z_{0}\right)-d\left(z_{0}, z\right) \geq \frac{\sin (\varepsilon)}{4} q_{0} r_{n} .
$$


There exists a constant $c_{1}>0$, such that

$$
c_{1}\left(\sin (\varepsilon) q_{0} r_{n} / 4\right)^{\alpha} \leq \mathcal{H}^{\alpha}\left(X \cap \bar{B}_{\sin (\varepsilon) q_{0} r_{n} / 4}\left(z_{0}\right)\right)
$$

for all $n \in \mathbb{N}$. Then

$$
\begin{aligned}
& \mathcal{M}_{p}^{\alpha}\left(X \cap B_{2 r_{n}}\left(z_{0}\right)\right) \\
& \quad \geq \int_{X \cap \bar{B}_{\sin (\varepsilon) q_{0} r_{n} / 4}\left(z_{0}\right)} \int_{\tilde{A}_{n}} \int_{\tilde{B}_{n}}\left(\frac{2 \operatorname{dist}\left(L_{x, y}, z\right)}{\|x-z\|\|y\|}\right)^{p} \mathrm{~d} \mathcal{H}^{\alpha}(x) \mathrm{d} \mathcal{H}^{\alpha}(y) \mathrm{d} \mathcal{H}^{\alpha}(z) \\
& \geq \int_{X \cap \bar{B}_{\sin (\varepsilon) q_{0} r_{n} / 4}\left(z_{0}\right)} \int_{\tilde{A}_{n}} \int_{\tilde{B}_{n}}\left(\frac{2 \frac{\sin (\varepsilon)}{4} q_{0} r_{n}}{4 r_{n}^{2}}\right)^{p} \mathrm{~d} \mathcal{H}^{\alpha}(x) \mathrm{d} \mathcal{H}^{\alpha}(y) \mathrm{d} \mathcal{H}^{\alpha}(z) \\
& \geq\left(\frac{\sin (\varepsilon) q_{0}}{8}\right)^{p} \mathcal{H}^{\alpha}\left(X \cap \bar{B}_{\sin (\varepsilon) q_{0} r_{n} / 4}\left(z_{0}\right)\right) \mathcal{H}^{\alpha}\left(\tilde{A}_{n}\right) \mathcal{H}^{\alpha}\left(\tilde{B}_{n}\right)\left(\frac{1}{r_{n}}\right)^{p} \\
& \geq\left(\frac{\sin (\varepsilon) q_{0}}{8}\right)^{p} c_{1}\left(\frac{\sin (\varepsilon) q_{0} r_{n}}{4}\right)^{\alpha} c^{2} r_{n}^{2 \alpha}\left(\frac{1}{r_{n}}\right)^{p} \geq\left(\frac{\sin (\varepsilon) q_{0}}{8}\right)^{p+\alpha} 2^{\alpha} c_{1} c^{2} r_{n}^{3 \alpha-p} \geq c^{\prime}>0
\end{aligned}
$$

for all $n \in \mathbb{N}$. Hence Lemma 2.11 tells us that $\mathcal{M}_{p}^{\alpha}(X)=\infty$, note that for this we needed $\mathcal{H}^{\alpha}\left(B_{2 r_{n}}(x) \cap X\right)<\infty$. This is absurd as we assumed $\mathcal{M}_{p}^{\alpha}(X)<\infty$.

Proposition 7.2 (Finite $\mathcal{M}_{p}^{\alpha}, p \geq 3 \alpha$ implies weak appr. tangents if $\Theta^{* \alpha}$ is finite). Let $X \subset \mathbb{R}^{n}$ be a set, $\alpha \in(0, \infty)$ and $x \in \mathbb{R}^{n}$ with $0<\Theta_{*}^{\alpha}\left(\mathcal{H}^{\alpha}, X, x\right) \leq \Theta^{* \alpha}\left(\mathcal{H}^{\alpha}, X, x\right)<$ $\infty$. If $p \in[3 \alpha, \infty)$ and $\mathcal{M}_{p}^{\alpha}(X)<\infty$ then $X$ has a weak approximate $\alpha$-tangent at $x$.

Proof. Assume that this is not the case. By Lemma 2.14 and Lemma 2.16 we can without loss of generality assume that $\mathcal{H}^{\alpha}\left(X \cap \bar{B}_{r}(x)\right)<\infty$ for all small radii. Then by Lemma 4.10 there is a mapping $s:(0, \rho) \rightarrow \mathbb{S}^{n-1}, \rho>0$ and $\varepsilon_{0}>0$, such that

$$
0<\Theta_{*}^{\alpha}\left(\mathcal{H}^{\alpha}, X \cap C_{s(r), \varepsilon_{0} / 2}(x), x\right)
$$

and

$$
0<\Theta^{* \alpha}\left(\mathcal{H}^{\alpha}, X \backslash C_{s(r), \varepsilon_{0}}(x), x\right) .
$$

This means that the hypotheses of Lemma 3.5 hold for

$$
A(r):=X \cap C_{s(r), \varepsilon_{0} / 2}(x) \text { and } B(r):=X \backslash C_{s(r), \varepsilon_{0}}(x),
$$

so that there exists a $q_{0} \in(0,1)$, a sequence $\left(r_{n}\right)_{n \in \mathbb{N}}, r_{n}>0, \lim _{n \rightarrow \infty} r_{n}=0$ and a constant $c>0$ such that

$$
\operatorname{cr}_{n}^{\alpha} \leq \min \left\{\mathcal{H}^{\alpha}\left(A\left(r_{n}\right) \cap\left[\bar{B}_{r_{n}}(x) \backslash B_{q_{0} r_{n}}(x)\right]\right), \mathcal{H}^{\alpha}\left(B\left(r_{n}\right) \cap\left[\bar{B}_{r_{n}}(x) \backslash B_{q_{0} r_{n}}(x)\right]\right)\right\} .
$$

Hence the hypotheses of Lemma 7.1 are fulfilled for $\varepsilon:=\varepsilon_{0} / 2$, note that $\mathcal{H}^{\alpha}\left(X \cap \bar{B}_{r}(x)\right)<$ $\infty$ for small radii, and we have proven the proposition.

\subsection{Finite $\mathcal{M}_{p}^{1}$ does not imply (weak) app. tangents for $p \in(0,3)$}

Definition 7.3 (The functional $\mathcal{F}_{p}$ ).

For $A, B, C \subset \mathbb{R}^{n}$ measurable, $p>0$ we set

$$
\mathcal{F}_{p}(A, B, C):=\int_{C} \int_{B} \int_{A} \kappa^{p}(x, y, z) \mathrm{d} \mathcal{H}^{1}(x) \mathrm{d} \mathcal{H}^{1}(y) \mathrm{d} \mathcal{H}^{1}(z)
$$




\section{Remark $7.4\left(\mathcal{F}_{p}\right.$ is invariant under permutations).}

By Fubini's Theorem and the symmetry of the integrand under permutations, as well as its measurability it is clear that for all measurable subsets $A, B, C \subset X$ of $X \subset \mathbb{R}^{n}$ we have

$$
\mathcal{F}_{p}(A, B, C)=\mathcal{F}_{p}(B, C, A)=\mathcal{F}_{p}(C, A, B)=\mathcal{F}_{p}(B, A, C)=\mathcal{F}_{p}(A, C, B)=\mathcal{F}_{p}(C, B, A) .
$$

Proposition 7.5 (The set $E$ has finite $\mathcal{M}_{p}^{1}$ for $p \in[2,3)$ ).

Let $E$ be the set from Definition 5.4. For $p \in[2,3)$ we have

$$
\mathcal{M}_{p}^{1}(E) \leq \frac{72 \pi}{(3-p)^{2}}
$$

Proof. Step 1 By Lemma 2.13 it is clear that

$$
\mathcal{M}_{p}^{1}(E)=\sum_{i, j, k \in\{1,2,3\}} \mathcal{F}_{p}\left(E_{i}, E_{j}, E_{k}\right)
$$

Since the integrand $\kappa^{p}$ vanishes on certain sets, we have

$$
\sum_{\substack{i, j, k \in\{1,2,3\} \\ \#\{i, j, k\}=1}} \mathcal{F}_{p}\left(E_{i}, E_{j}, E_{k}\right)+\sum_{i, j, k \in\{1,3\}} \mathcal{F}_{p}\left(E_{i}, E_{j}, E_{k}\right)=0
$$

furthermore

$$
\mathcal{M}_{p}^{1}\left(E_{1} \cup E_{2}\right)=\sum_{\substack{i, j, k \in\{1,2\} \\ \#\{i, j, k\}=2}} \mathcal{F}_{p}\left(E_{i}, E_{j}, E_{k}\right)=\sum_{\substack{i, j, k \in\{2,3\} \\ \#\{i, j, k\}=2}} \mathcal{F}_{p}\left(E_{i}, E_{j}, E_{k}\right)=\mathcal{M}_{p}^{1}\left(E_{2} \cup E_{3}\right),
$$

as the energy is invariant under isometries. Considering Remark 7.4 we obtain

$$
\mathcal{M}_{p}^{1}\left(E_{1} \cup E_{2}\right)=\mathcal{M}_{p}^{1}\left(E_{2} \cup E_{3}\right)=3\left(\mathcal{F}_{p}\left(E_{1}, E_{1}, E_{2}\right)+\mathcal{F}_{p}\left(E_{1}, E_{2}, E_{2}\right)\right)=6 \mathcal{F}_{p}\left(E_{1}, E_{1}, E_{2}\right),
$$

where the last equality is, again, due to the invariance of the integrand under isometries. By considering the integrand $\kappa^{p}$ in the form

$$
\kappa^{p}(x, y, z)=\left(\frac{2 \operatorname{dist}\left(x, L_{z y}\right)}{d(x, y) d(x, z)}\right)^{p}
$$

for $x \in E_{2}, y \in E_{1}$ and $z \in E_{3}$ we note, that $\kappa^{p}(x, y, z)=\kappa^{p}(x, y,-z)$, by mapping $E_{3}$ onto $E_{1}$ via $z \mapsto-z$ we find

$$
\mathcal{F}_{p}\left(E_{3}, E_{1}, E_{2}\right)=\mathcal{F}_{p}\left(E_{1}, E_{1}, E_{2}\right),
$$

so that

$$
\sum_{\substack{i, j, k \in\{1,2,3\} \\ \#\{i, j, k\}=3}} \mathcal{F}_{p}\left(E_{i}, E_{j}, E_{k}\right)=6 \mathcal{F}_{p}\left(E_{1}, E_{1}, E_{2}\right)
$$


All in all we obtain

$$
\begin{aligned}
& \mathcal{M}_{p}^{1}(E) \\
& =\left(\sum_{\substack{i, j, k \in\{1,2,3\} \\
\#\{i, j, k\}=1}}+\sum_{\substack{i, j, k \in\{1,3\} \\
\#\{i, j, k\}=2}}+\sum_{\substack{i, j, k \in\{1,2\} \\
\#\{i, j, k\}=2}}+\sum_{\substack{i, j, k \in\{2,3\} \\
\#\{i, j, k\}=2}}+\sum_{\substack{i, j, k \in\{1,2,3\} \\
\#\{i, j, k\}=3}}\right) \mathcal{F}_{p}\left(E_{i}, E_{j}, E_{k}\right) \\
& =18 \mathcal{F}_{p}\left(E_{1}, E_{1}, E_{2}\right)=18 \mathcal{F}_{p}\left(E_{2}, E_{1}, E_{1}\right) \text {. }
\end{aligned}
$$

Step 2 Let us first choose parametrisations

$$
\gamma_{1}:[0,1] \rightarrow \mathbb{R}^{2}, t \mapsto(-t, 0) \quad \text { and } \quad \gamma_{2}:[0,1] \rightarrow \mathbb{R}^{2}, t \mapsto(0, t)
$$

of $E_{1}$ and $E_{2}$, respectively. This gives us

$$
\begin{aligned}
& \mathcal{F}_{p}\left(E_{2}, E_{1}, E_{1}\right)=\int_{0}^{1} \int_{0}^{1} \int_{0}^{1}\left(\frac{2 x}{\sqrt{x^{2}+y^{2}} \sqrt{x^{2}+z^{2}}}\right)^{p} \mathrm{~d} \mathcal{L}^{1}(x) \mathrm{d} \mathcal{L}^{1}(y) \mathrm{d} \mathcal{L}^{1}(z) \\
& \stackrel{\text { Lemma C.2 }}{\leq} \int_{0}^{1} \int_{0}^{1} 2^{p} \frac{\pi}{2^{p}}(z y)^{-(p-1) / 2} \mathrm{~d} \mathcal{L}^{1}(y) \mathrm{d} \mathcal{L}^{1}(z) \\
& \quad=\pi \int_{0}^{1} z^{(1-p) / 2}\left[\frac{2}{3-p} y^{(3-p) / 2}\right]_{0}^{1} \mathrm{~d} \mathcal{L}^{1}(z)=\pi\left[\frac{2}{3-p} z^{(3-p) / 2}\right]_{0}^{1} \frac{2}{3-p} \\
& \quad=\frac{4 \pi}{(3-p)^{2}}
\end{aligned}
$$

Notice that the range $p \geq 2$ was neccessary to apply Lemma C.2.

Corollary 7.6 (The set $E$ has finite $\mathcal{M}_{p}^{1}$ for $\left.p \in(0,3)\right)$.

For $p \in(0,3)$ we have $\mathcal{M}_{p}^{1}(E)<\infty$.

Proof. This is a consequence of Lemma 7.5 together with $\mathcal{H}^{1}(E)=3$ and Lemma 2.10.

\section{Exponents are sharp and weak approximate tangents are optimal for $\alpha=1$}

The exponents in the previous results are sharp, i.e.

Lemma 8.1 (A set with no appr. 1-tangent and finite $\mathcal{U}_{(0,1)}^{1}, \mathcal{I}_{(0,2)}^{1}$ and $\mathcal{M}_{(0,3)}^{1}$ ). Let $E$ be the set from Definition 5.4. Then

- E does not have a weak approximate 1-tangent at 0 ,

- $\mathcal{U}_{p}^{1}(E)<\infty$ for all $p \in(0,1)$,

- $\mathcal{I}_{p}^{1}(E)<\infty$ for all $p \in(0,2)$,

- $\mathcal{M}_{p}^{1}(E)<\infty$ for all $p \in(0,3)$.

Proof. This is Lemma 5.5, Corollary 6.4 and Corollary 7.6.

The weak approximate 1 -tangents in the results for $\mathcal{I}_{p}^{1}$ and $\mathcal{M}_{p}^{1}$ are optimal in the following sense 
Lemma 8.2 (A set with no appr. tangent and finite $\mathcal{I}_{p}^{1}$ for all $p \in(0, \infty)$ ). Set $a_{n}:=2^{-n^{n} n^{3}}, A_{n}:=\left[a_{n} / 2, a_{n}\right]$ and

$$
F:=[\bigcup_{n \in \mathbb{N}} \underbrace{A_{2 n} \times\{0\}}_{=: B_{2 n}}] \cup\left[\bigcup_{n \in \mathbb{N}}^{\{0\} \times A_{2 n-1}}\right] .
$$

Then

- F does not have an approximate 1 -tangent at 0 ,

- $1 / \Delta[F]=\infty$,

- $\mathcal{U}_{p}^{1}(F)=\infty$ for all $p \in[1, \infty)$,

- $\mathcal{I}_{p}^{1}(F)<\infty$ for all $p \in(0, \infty)$,

- $\mathcal{M}_{p}^{1}(F)<\infty$ for all $p \in(0, \infty)$.

Proof. Step 1 For $l \neq k$ we denote $\mu:=\min \{k, l\}$ and $M:=\max \{k, l\}$. Then

$$
\begin{aligned}
& \operatorname{dist}\left(B_{k}, B_{l}\right) \geq \operatorname{dist}\left(A_{k}, A_{l}\right)=2^{-\left(\mu^{\mu} \mu^{3}+1\right)}-2^{-M^{M} M^{3}} \\
& \quad=2^{-\left(\mu^{\mu} \mu^{3}+1\right)}\left(1-2^{\left(\mu^{\mu} \mu^{3}+1\right)-M^{M} M^{3}}\right) \geq 2^{-\left(\mu^{\mu} \mu^{3}+2\right)}=a_{\mu} / 4 .
\end{aligned}
$$

Let $y \in B_{k}, z \in B_{l}$ with $k \neq l$. Then

$$
\kappa_{i}(y, z) \leq \frac{2}{\operatorname{dist}\left(B_{k}, B_{l}\right)} \leq \frac{8}{a_{\mu}}=\frac{8}{a_{\min \{k, l\}}}=\frac{8}{\max \left\{a_{k}, a_{l}\right\}} .
$$

Step 2 Let $q>1$. We now compute some inequalities for the indices. Let $k, m \in \mathbb{N}$, $k<m$, i.e. $m=k+i$ for some $i \in \mathbb{N}$. Then

$$
m^{3}=(k+i)^{3}=k^{3}+3 k^{2} i+3 k i^{2}+i^{3},
$$

so that

$$
-m^{3}+k^{3}=-\left(3 k^{2} i+3 k i^{2}+i^{3}\right) \leq-3(k+i)=-3 m .
$$

As $q k^{k} \leq m^{m}$ for $1<q \leq k<m$ we have

$$
-m^{m} m^{3}+q k^{k} k^{3} \leq-q k^{k} m^{3}+q k^{k} k^{3}=q k^{k}\left(-m^{3}+k^{3}\right) \stackrel{(11)}{\leq} q k^{k}(-3 m) \leq-3 m .
$$

Consequently for all $1<q \leq k<m$

$$
\frac{a_{m}}{a_{k}^{q}}=\frac{2^{-m^{m} m^{3}}}{2^{-q k^{k} k^{3}}}=2^{-m^{m} m^{3}+q k^{k} k^{3}} \leq 2^{-3 m} .
$$


Step 3 As $\mathcal{H}^{1}\left(B_{n}\right)=a_{n} / 2$ we have for $p \geq 3$, and $q=p-1>1$

$$
\begin{aligned}
& \sum_{\substack{k, m \in \mathbb{N} \\
k \neq m}} \int_{B_{k}} \int_{B_{m}} \kappa_{i}^{p}(y, z) \mathrm{d} \mathcal{H}^{1}(y) \mathrm{d} \mathcal{H}^{1}(z) \\
& \leq \sum_{\substack{k, m \in \mathbb{N} \\
k \neq m}}\left[\frac{8}{\max \left\{a_{k}, a_{m}\right\}}\right]^{p} \frac{a_{k} a_{m}}{4} \\
& \leq \frac{2 \cdot 8^{p}}{4} \sum_{\substack{k, m \in \mathbb{N} \\
1 \leq k<m}} \frac{a_{k} a_{m}}{\max \left\{a_{k}, a_{m}\right\}^{p}} \\
& \leq 4 \cdot 8^{p-1} \sum_{\substack{1 \leq k \leq q \\
k<m}} \frac{a_{k} a_{m}}{\max \left\{a_{k}, a_{m}\right\}^{p}}+4 \cdot 8^{p-1} \sum_{\substack{k, m \in \mathbb{N} \\
q \leq k<m}} \frac{a_{m}}{a_{k}^{p-1}} \\
& \leq 4 \cdot 8^{p-1} \sum_{\substack{1 \leq k \leq q \\
k<m}} \frac{a_{k} a_{m}}{a_{\lceil q\rceil}^{p}}+4 \cdot 8^{p-1} \sum_{\substack{k, m \in \mathbb{N} \\
q \leq k<m}} \frac{a_{m}}{a_{k}^{q}} \\
& \stackrel{(12)}{\leq} \frac{4 \cdot 8^{p-1}}{a_{\lceil q\rceil}^{p}} \sum_{k, m \in \mathbb{N}} 2^{-k} 2^{-m}+4 \cdot 8^{p-1} \sum_{\substack{k, m \in \mathbb{N} \\
q \leq k<m}} 2^{-3 m} \\
& \leq \frac{4 \cdot 8^{p-1}}{a_{\lceil q\rceil}^{p}}+4 \cdot 8^{p-1} \sum_{\substack{k, m \in \mathbb{N} \\
q \leq k<m}} 2^{-k} 2^{-m} \\
& \leq \frac{4 \cdot 8^{p-1}}{a_{\lceil q\rceil}^{p}}+4 \cdot 8^{p-1} \sum_{k, m \in \mathbb{N}} 2^{-k} 2^{-m} \\
& =4 \cdot 8^{p-1}\left(\frac{1}{a_{\lceil q\rceil}^{p}}+1\right) \text {. }
\end{aligned}
$$

Step 4 Let $y, z \in B_{n}$. Then $\kappa(x, y, z)>0$ if and only if $x \in B_{k}$ for $(k-n) \bmod 2=1$. To simplify matters we may without loss of generality assume that $k$ is even and $n$ is odd. We now have, compare Remark 2.2,

$$
\kappa(x, y, z)=\frac{2 \xi}{\sqrt{\xi^{2}+\eta^{2}} \sqrt{\xi^{2}+\zeta^{2}}},
$$

where we denote the non-zero entries of $x, y$ and $z$ by $\xi, \eta$ and $\zeta$, respectively. If we set $f(\xi):=\kappa(x, y, z) / 2$ for fixed $y$ and $z$ we have

$$
\begin{aligned}
f^{\prime}(\xi) & =\frac{1}{{\sqrt{\xi^{2}+\eta^{2}}}^{\xi^{2}+\zeta^{2}}}-\frac{\xi^{2}}{{\sqrt{\xi^{2}+\eta^{2}}}^{3}{\sqrt{\xi^{2}+\zeta^{2}}}^{2}}-\frac{\xi^{2}}{\sqrt{\xi^{2}+\eta^{2}}{\sqrt{\xi^{2}+\zeta^{2}}}^{3}} \\
& =\frac{\left(\xi^{2}+\eta^{2}\right)\left(\xi^{2}+\zeta^{2}\right)}{{\sqrt{\xi^{2}+\eta^{2}}}^{3}{\sqrt{\xi^{2}+\zeta^{2}}}^{3}}-\frac{\xi^{2}\left(\xi^{2}+\zeta^{2}\right)+\xi^{2}\left(\xi^{2}+\eta^{2}\right)}{{\sqrt{\xi^{2}+\eta^{2}}}^{3}{\sqrt{\xi^{2}+\zeta^{2}}}^{3}} \\
& =\frac{\left(\xi^{2}+\eta^{2}\right) \zeta^{2}-\xi^{2}\left(\xi^{2}+\zeta^{2}\right)}{{\sqrt{\xi^{2}+\eta^{2}}}^{3}{\sqrt{\xi^{2}+\zeta^{2}}}^{3}}=\frac{\eta^{2} \zeta^{2}-\xi^{4}}{{\sqrt{\xi^{2}+\eta^{2}}}^{3}{\sqrt{\xi^{2}+\zeta^{2}}}^{3}},
\end{aligned}
$$

which is 0 if and only if $\xi=\sqrt{\eta \zeta}$, because $\xi, \eta, \zeta>0$. That $f$ attains its maximum at $\xi=\sqrt{\eta \zeta}$ is clear by $f^{\prime} \geq 0$ on $[0, \sqrt{\eta \zeta}]$ and $f^{\prime} \leq 0$ on $[\sqrt{\eta \zeta}, \infty)$. Since $\sqrt{\eta \zeta} \in A_{n}$ we have 
$(\sqrt{\eta \zeta}, 0) \notin F$, as $n$ is odd, so that $\kappa_{i}(y, z)=\sup _{x \in F} \kappa(x, y, z)$ is attained for $x=(\xi, 0)$, $\xi \in\left\{a_{n+1}, a_{n-1} / 2\right\}$. We have

$$
f\left(a_{n+1}\right)=\frac{a_{n+1}}{\sqrt{a_{n+1}^{2}+\eta^{2}} \sqrt{a_{n+1}^{2}+\zeta^{2}}} \leq \frac{a_{n+1}}{a_{n+1}^{2}+a_{n}^{2} / 4} \leq 4 \frac{a_{n+1}}{a_{n}^{2}}
$$

and

$$
f\left(a_{n-1} / 2\right)=\frac{a_{n-1} / 2}{\sqrt{a_{n-1}^{2} / 4+\eta^{2}} \sqrt{a_{n-1}^{2} / 4+\zeta^{2}}} \leq \frac{a_{n-1} / 2}{a_{n-1}^{2} / 4+a_{n}^{2} / 4} \leq 2 \frac{a_{n-1}}{a_{n-1}^{2}} \leq \frac{4}{a_{n-1}} .
$$

As $2 n^{n} n^{3} \leq(n+1)(n+1)^{n}(n+1)^{3}=(n+1)^{n+1}(n+1)^{3}$ and $a_{n-1} \leq 1$ we have $a_{n+1} a_{n-1} \leq a_{n}^{2}$ and hence for $n \geq 2$

$$
\kappa_{i}(y, z)=2 \max \left\{f\left(a_{n+1}\right), f\left(a_{n-1} / 2\right)\right\} \leq 2 \max \left\{\frac{4 a_{n+1}}{a_{n}^{2}}, \frac{4}{a_{n-1}}\right\}=\frac{8}{a_{n-1}} .
$$

Consequently we have for $p \geq 3$

$$
\begin{aligned}
& \sum_{n=1}^{\infty} \int_{B_{n}} \int_{B_{n}} \kappa_{i}^{p}(y, z) \mathrm{d} \mathcal{H}^{1}(y) \mathrm{d} \mathcal{H}^{1}(z) \\
& \quad \leq \frac{2^{p}}{\operatorname{dist}\left(B_{1}, \mathbb{R} \times\{0\}\right)^{p}}\left(\frac{1}{8}\right)^{2}+\sum_{n=2}^{\infty} \frac{8^{p}}{a_{n-1}^{p}} \mathcal{H}^{1}\left(B_{n}\right) \mathcal{H}^{1}\left(B_{n}\right) \\
& \quad \leq \frac{2^{p}}{(1 / 4)^{p}}\left(\frac{1}{8}\right)^{2}+\sum_{n=2}^{\infty} \frac{8^{p}}{a_{n-1}^{p}} \frac{a_{n}^{2}}{4} \leq \frac{8^{p}}{64}+8^{p} \sum_{n=2}^{\infty} \frac{a_{n}}{a_{n-1}^{p}} \\
& \quad \leq \frac{8^{p}}{64}+8^{p} \sum_{n=2}^{\lceil p\rceil+1} \frac{a_{n}}{a_{n-1}^{p}}+8^{p} \sum_{n=\lceil p\rceil+1}^{\infty} \frac{a_{n}}{a_{n-1}^{p}} \\
& \quad \leq C_{p}+8^{p} \sum_{n=\lceil p\rceil+1}^{\infty} 2^{-3 n} \leq C_{p}+8^{p} \sum_{n=0}^{\infty} 2^{-n} \leq C_{p}+8^{p} \cdot 2 .
\end{aligned}
$$

Step 5 For $p \geq 3$ we now conclude that by Lemma 2.13 we have

$$
\begin{aligned}
\mathcal{I}_{p}^{1}(F) & \leq \sum_{k, l \in \mathbb{N}} \int_{B_{k}} \int_{B_{l}} \kappa_{i}^{p}(y, z) \mathrm{d} \mathcal{H}^{1}(y) \mathrm{d} \mathcal{H}^{1}(z) \\
& =\sum_{\substack{k, l \in \mathbb{N} \\
k \neq l}} \int_{B_{k}} \int_{B_{l}} \kappa_{i}^{p}(y, z) \mathrm{d} \mathcal{H}^{1}(y) \mathrm{d} \mathcal{H}^{1}(z)+\sum_{n \in \mathbb{N}} \int_{B_{n}} \int_{B_{n}} \kappa_{i}^{p}(y, z) \mathrm{d} \mathcal{H}^{1}(y) \mathrm{d} \mathcal{H}^{1}(z)<\infty
\end{aligned}
$$

Using $\mathcal{H}^{1}(F) \leq 2$ together with Lemma 2.10 we have $\mathcal{I}_{p}^{1}(F)<\infty$ for all $p \in(0, \infty)$.

Step 6 In Example 4.7 we have already seen that $F$ has no approximate tangent at 0 . This observation combined with Lemma 5.1 directly gives us $1 / \Delta[F]=\infty$ and $\mathcal{U}_{p}^{1}(F)=\infty$ for all $p \in[1, \infty)$. For $\mathcal{M}_{p}^{1}(F)<\infty$ for all $p \in(0, \infty)$ we consult Lemma 2.9 together with $\mathcal{H}^{1}(F) \leq 2$.

\section{A Semi-continuous and measurable functions}

Lemma A.1 (Metric is continuous).

The mapping $f: X^{3} \rightarrow \mathbb{R},(x, y, z) \mapsto d(x, y)$ is continuous. 
Proof. A mapping from a metric space to a metric space is continuous iff it is sequentially continuous. Let $(x, y, z) \in X^{3}$ and

$$
\left(x_{n}, y_{n}, z_{n}\right) \underset{n \rightarrow \infty}{\stackrel{\left(X^{3}, d^{3}\right)}{\longrightarrow}}(x, y, z) .
$$

Then $x_{n} \rightarrow x$ and $y_{n} \rightarrow y$ in $X$ for $n \rightarrow \infty$, which gives us

$$
\begin{aligned}
\left|d(x, y)-d\left(x_{n}, y_{n}\right)\right| & \leq\left|d(x, y)-d\left(x_{n}, y\right)\right|+\left|d\left(x_{n}, y\right)-d\left(x_{n}, y_{n}\right)\right| \\
& \leq d\left(x, x_{n}\right)+d\left(y, y_{n}\right) \underset{n \rightarrow \infty}{\longrightarrow} 0 .
\end{aligned}
$$

\section{Lemma A.2 (Reciprocal of semi-continuous functions).}

Let $f:(X, d) \rightarrow \overline{\mathbb{R}}, f \geq 0$ be lower [upper] semi-continuous then $1 / f$ is upper [lower] semi-continuous, if we set $1 / 0=\infty$ and $1 / \infty=0$.

Proof. A function $f$ is lower semi-continuous if and only if for all $t \in \mathbb{R}$ the set $\{f \leq t\}$ is closed, see [Bra02, Remark 1.3, p.21]. Hence the sets $\{1 / t \leq 1 / f\}$ are closed for $t>0$, as is $\{\alpha \leq 1 / f\}=X$ for $\alpha \leq 0$. The other case follows analogously.

\section{Lemma A.3 (Semi-continuous functions are measurable).}

Let $f:(X, d) \rightarrow \overline{\mathbb{R}}$ be upper or lower semi-continuous then $f$ is $\mathcal{B}(X)-\mathcal{B}(\overline{\mathbb{R}})$ measurable.

Proof. If $f$ is lower semi-continuous then the set $\{f \leq t\}$ is closed for all $t \in \mathbb{R}$ and if $f$ is upper semi-continuous then the set $\{t \leq f\}$ is closed and hence a Borel set.

Lemma A.4 (Positive powers of positive, s.c. functions are s.c.).

Let $f:(X, d) \rightarrow \overline{\mathbb{R}}, f \geq 0$ be lower [upper] semi-continuous then for all $p \in(0, \infty)$ the function $f^{p}$ is lower [upper] semicontinuous.

Proof. Without loss of generality let $f \geq 0$ be lower semi-continuous. We have $\{f \leq t\}=$ $\emptyset$ for $t<0$. If $t \geq 0$ we clearly have $f(x)^{p} \leq t \Leftrightarrow f(x) \leq t^{1 / p}$.

Lemma A.5 (Measurability of piecewise functions).

Let $\left(X_{1}, \mathcal{A}_{1}\right),\left(X_{2}, \mathcal{A}_{2}\right)$ be measuring spaces, $A \in \mathcal{A}_{1}$ and $f: A \rightarrow X_{2}$ be $\left.\mathcal{A}_{1}\right|_{A}-\mathcal{A}_{2}$ measurable and $g: X_{1} \backslash A \rightarrow X_{2}$ be $\left.\mathcal{A}_{1}\right|_{X_{1} \backslash A}-\mathcal{A}_{2}$ measurable. Then

$$
F: X_{1} \rightarrow X_{2}, x \mapsto \begin{cases}f(x), & x \in A, \\ g(x), & x \in X_{1} \backslash A,\end{cases}
$$

is $\mathcal{A}_{1}-\mathcal{A}_{2}$ measurable.

Proof. Let $E \in \mathcal{A}_{2}$, then there exist measurable sets $B, C \in \mathcal{A}_{1}$ such that

$$
F^{-1}(E)=f^{-1}(E) \cup g^{-1}(E)=(B \cap A) \cup\left(C \cap\left[X_{1} \backslash A\right]\right) \stackrel{A \in \mathcal{A}_{1}}{\in} \mathcal{A}_{1} .
$$

Lemma A.6 (Extension of lower semi-continuous functions).

Let $(X, d)$ be a metric space, $C \subset X$ closed and $f: X \backslash C \rightarrow \overline{\mathbb{R}}, f \geq 0$ lower semicontinuous. Then

$$
\tilde{f}: X \rightarrow \overline{\mathbb{R}}, x \mapsto \begin{cases}f(x), & x \in X \backslash C, \\ 0, & x \in C,\end{cases}
$$

is lower semi continuous. 
Proof. Let $\left(x_{n}\right)_{n \in \mathbb{N}} \subset X$ be a sequence converging to $x \in X$. If $x_{n} \in C$ for infinitely many $n \in \mathbb{N}$ the sequence with these indices is contained in $C$ and converges to $x$, so that, since $C$ is closed, we have $x \in C$ and consequently

$$
\tilde{f}(x)=0 \leq \liminf _{n \rightarrow \infty} \tilde{f}\left(x_{n}\right) .
$$

If $x_{n} \in C$ only for a finite number of $n \in \mathbb{N}$, we can use the lower semi-continuity of $f$ on $X \backslash C$ to get

$$
\tilde{f}(x)=f(x) \leq \liminf _{n \rightarrow \infty} f\left(x_{n}\right)=\liminf _{n \rightarrow \infty} \tilde{f}\left(x_{n}\right) \quad \text { if } x \in X \backslash C
$$

and

$$
\tilde{f}(x)=0 \leq \liminf _{n \rightarrow \infty} \underbrace{\tilde{f}\left(x_{n}\right)}_{\geq 0} \quad \text { if } x \in C .
$$

Lemma A.7 ( $\operatorname{diag}(X)$ and $X_{0}$ are closed).

Let $(X, d)$ be a metric space. Then the diagonal $\operatorname{diag}(X)$ and $X_{0}$ are closed sets.

Proof. Step 1 Let $\left(\left(x_{n}, y_{n}\right)\right)_{n \in \mathbb{N}} \subset \operatorname{diag}(X)$ be a sequence converging to $(x, y) \in X^{2}$. Then $x_{n}=y_{n}$ and since convergence in the product space implies convergence of the projections we have $x_{n}=y_{n} \rightarrow x=y$, where we have used, that in Hausdorff spaces limits are unique.

Step 2 The set $X_{0}$ is closed in the product space, because let $\left(\left(x_{n}, y_{n}, z_{n}\right)\right)_{n \in \mathbb{N}} \subset X_{0}$ be a sequence converging to $(x, y, z) \in X^{3}$. Since $X_{0}$ is the union of the three sets

$$
\operatorname{diag}(X) \times X, \quad\left\{(x, y, x) \in X^{3} \mid x, y \in X\right\} \quad \text { and } \quad X \times \operatorname{diag}(X)
$$

there exists a subsequence converging to the same limit, which is contained in one of these sets. Clearly these sets are closed, so that $X_{0}$ is closed.

Lemma A.8 (Cauchy sequence in complete metric spaces). Let $(X, d)$ be a complete metric space, $\left(x_{n}\right)_{n \in \mathbb{N}} \subset X$ with

$$
d\left(x_{n}, x_{n+1}\right) \leq a_{n} \quad \text { and } \quad \sum_{n=1}^{\infty} a_{n}<\infty .
$$

Then there is $x \in X$, such that $x_{n} \rightarrow x$ and

$$
d\left(x_{n}, x\right) \leq \sum_{i=n}^{\infty} a_{i}
$$

Proof. Let $\varepsilon>0$ and $N$ be large enough for $\sum_{i=N}^{\infty} a_{i} \leq \varepsilon$, which is possible, as $\sum_{i=N}^{\infty} a_{i} \rightarrow$ 0 . Let $m, n \geq N$, without loss of generality $m>n$. Then

$$
d\left(x_{n}, x_{m}\right) \leq \sum_{i=n}^{m-1} d\left(x_{i}, x_{i+1}\right) \leq \sum_{i=n}^{m-1} a_{i} \leq \sum_{i=n}^{\infty} a_{i} \leq \varepsilon
$$


so that $\left(x_{n}\right)_{n \in \mathbb{N}}$ is a Cauchy sequence in the complete space $(X, d)$ and hence convergent. This means there is $x \in X$, such that $x_{n} \rightarrow x$. Then for all $N>n$ we have

$$
d\left(x_{n}, x\right) \leq \sum_{i=n}^{N-1} d\left(x_{i}, x_{i+1}\right)+d\left(x_{N}, x\right) \leq \sum_{i=n}^{N-1} a_{i}+d\left(x_{N}, x\right)
$$

and hence

$$
d\left(x_{n}, x\right)=\lim _{N \rightarrow \infty} d\left(x_{n}, x\right) \leq \lim _{N \rightarrow \infty}\left[\sum_{i=n}^{N-1} a_{i}+d\left(x_{N}, x\right)\right]=\sum_{i=n}^{\infty} a_{i} .
$$

\section{B Curvature energies under removal of acc. point}

Lemma B.1 ( $\kappa_{i}$ if accumulation point is removed).

Let $(X, d)$ be a metric space and $x \in X$. If $x$ is an accumulation point of $X$ then

$$
\kappa_{i}^{X}(y, z)=\kappa_{i}^{X \backslash\{x\}}(y, z), \quad \text { for all } y, z \in X \backslash\{x\}, y \neq z .
$$

Proof. Let $y, z \in X \backslash\{x\}, y \neq z$. Then there is a sequence $\left(x_{n}\right)_{n \in \mathbb{N}} \subset X \backslash\{y, z\}$, such that

$$
\frac{1}{r\left(x_{n}, y, z\right)} \underset{n \rightarrow \infty}{\longrightarrow} \kappa_{i}(y, z) \text {. }
$$

If there is a subsequence $x_{n_{k}} \neq x$ for all $k \in \mathbb{N}$ the proposition is clear, so we assume $x_{n}=x$ for all $n \geq N$. We then find a sequence $\bar{x}_{n} \in X \backslash\{x, y, z\}$, such that $\bar{x}_{n} \rightarrow x$ and as $r$ is continuous, see Lemma 2.3 (i), this gives us

$$
\frac{1}{r\left(\bar{x}_{n}, y, z\right)} \underset{n \rightarrow \infty}{\longrightarrow} \frac{1}{r(x, y, z)}=\kappa_{i}(y, z)
$$

Lemma B.2 ( $\kappa_{G}$ if accumulation point is removed).

Let $(X, d)$ be a metric space and $x \in X$. If $x$ is an accumulation point of $X$ then

$$
\kappa_{G}^{X}(z)=\kappa_{G}^{X \backslash\{x\}}(z), \quad \text { for all } z \in X \backslash\{x\} .
$$

Proof. We may without loss of generality assume that $\# X \geq 3$, as otherwise $\kappa_{G} \equiv 0$ and $\kappa_{i} \equiv 0$ for both $X$ and $X \backslash\{x\}$. Let $z \in X \backslash\{x\}$ then there are sequences $\left(x_{n}\right)_{n \in \mathbb{N}}$ and $\left(y_{n}\right)_{n \in \mathbb{N}}$ in $X \backslash\{z\}$ with $x_{n} \neq y_{n}$ for all $n \in \mathbb{N}$, such that

$$
\frac{1}{r\left(x_{n}, y_{n}, z\right)} \rightarrow \kappa_{G}(z)
$$

If there is a subsequence $\left(n_{k}\right)_{k \in \mathbb{N}}$, such that $x_{n_{k}}, y_{n_{k}} \neq x$ the proposition is clear. Let $x_{n_{l}}=x$ for all $l \in \mathbb{N}$. For fixed $l$ there exists a sequence $\left(x_{k}^{l}\right)_{k \in \mathbb{N}}$ with $x_{k}^{l} \rightarrow x, k \rightarrow \infty$, such that $x_{k}^{l} \notin\left\{x, y_{n_{l}}, z\right\}$. As $r$ is continuous, see Lemma 2.3 (i), and $\#\left\{x_{k}^{l}, y_{n_{l}}, z\right\}=3$ we have

$$
\frac{1}{r\left(x_{k}^{l}, y_{n_{l}}, z\right)} \underset{k \rightarrow \infty}{\longrightarrow} \frac{1}{r\left(x, y_{n_{l}}, z\right)} \text {. }
$$


Case 1 Assume $\kappa_{G}(z)<\infty$. Then for all $\varepsilon>0$ there exists $l \in \mathbb{N}$ and $k \in \mathbb{N}$, such that

$$
\begin{aligned}
& \left|\kappa_{G}(z)-\frac{1}{r\left(x_{k}^{l}, y_{n_{l}}, z\right)}\right| \leq\left|\kappa_{G}(z)-\frac{1}{r\left(x, y_{n_{l}}, z\right)}\right|+\left|\frac{1}{r\left(x, y_{n_{l}}, z\right)}-\frac{1}{r\left(x_{k}^{l}, y_{n_{l}}, z\right)}\right| \\
& \leq \varepsilon+\varepsilon=2 \varepsilon .
\end{aligned}
$$

Case 2 Assume $\kappa_{G}(z)=\infty$. If there is an $l$, such that $1 / r\left(x, y_{n_{l}}, z\right)=\infty$, then the proposition is clear by (14). We therefore assume that $1 / r\left(x, y_{n_{l}}, z\right)<\infty$ for all $l \in \mathbb{N}$. Then there exists a $K_{l}>0$ such that

$$
\left|\frac{1}{r\left(x, y_{n_{l}}, z\right)}-\frac{1}{r\left(x_{k}^{l}, y_{n_{l}}, z\right)}\right| \leq 1 \quad \text { for all } k \geq K_{l} .
$$

Furthermore for all $M>0$ there is a $L_{M}>0$, such that

$$
M \leq \frac{1}{r\left(x, y_{n_{l}}, z\right)} \quad \text { for all } l \geq L_{M}
$$

Hence for all $M>1$ there are $l_{0}$ and $k_{0}$, such that

$$
\begin{aligned}
M- & \leq\left|\frac{1}{r\left(x, y_{n_{l}}, z\right)}\right|-\left|\frac{1}{r\left(x, y_{n_{l}}, z\right)}-\frac{1}{r\left(x_{k}^{l}, y_{n_{l}}, z\right)}\right| \\
& \leq \frac{1}{r\left(x_{k}^{l}, y_{n_{l}}, z\right)}
\end{aligned}
$$

so that $\kappa_{G}^{X \backslash\{x\}}(z)=\infty$.

Lemma B.3 ( $\mathcal{F}$ if accumulation point is removed).

Let $(X, d)$ be a metric space, $x \in X, \alpha \in(0, \infty), p \in(0, \infty)$ and $\mathcal{F} \in\left\{\mathcal{U}_{p}^{\alpha}, \mathcal{I}_{p}^{\alpha}, \mathcal{M}_{p}^{\alpha}\right\}$. If $x$ is an accumulation point of $X$ then

$$
\mathcal{F}(X)=\mathcal{F}(X \backslash\{x\}) .
$$

Proof. For all metric spaces we have $\mathcal{H}^{\alpha}(\{x\})=0$, because for all $\varepsilon>0$ we can cover $\{x\}$ with $B_{\varepsilon}(x)$, which has diameter $2 \varepsilon$. From the definition of the integral it is now clear that for $\mathcal{H}^{\alpha}$ integration we can neglect singletons, hence together with Lemma B.1 and Lemma B.2 we have $\mathcal{F}(X)=\mathcal{F}(X \backslash\{x\})$ for all $p \in(0, \infty)$, if we recall that by Lemma B.4 we also have $\mathcal{H}_{X \backslash\{x\}}^{\alpha}=\left.\mathcal{H}^{\alpha}\right|_{X \backslash\{x\}}$.

\section{Lemma B.4 (Hausdorff measure on subspaces).}

Let $(X, d)$ be a metric space $A \subset X$ and $\left(A, d_{A}\right)$ the associated metric subspace. Then for all $\alpha>0$

$$
\mathcal{H}_{\left(A, d_{A}\right)}^{\alpha}=\mathcal{H}_{(X, d)}^{\alpha} \mid A
$$

Proof. Let $M \subset A$.

Step 1 Let $\left(C_{n}\right)_{n \in \mathbb{N}}$ be a $\delta$-covering of $M$ in $(X, d)$, then $\left(D_{n}\right)_{n \in \mathbb{N}}$ defined by $D_{n}:=C_{n} \cap A$ is a $\delta$-covering of $M$ in $\left(A, d_{A}\right)$, such that

$$
\operatorname{diam}_{A}\left(D_{n}\right) \leq \operatorname{diam}_{X}\left(C_{n}\right) \text { for all } n \in \mathbb{N},
$$


which implies

$$
\mathcal{H}_{A, \delta}^{\alpha}(M) \leq \mathcal{H}_{X, \delta}^{\alpha}(M)
$$

and thereby " $\leq$ ".

Step 2 Let $\left(D_{n}\right)_{n \in \mathbb{N}}$ be a $\delta$-covering of $M$ in $\left(A, d_{A}\right)$, then $\left(D_{n}\right)_{n \in \mathbb{N}}$ is also a $\delta$-covering of $M$ in $(X, d)$, which gives us

$$
\mathcal{H}_{X, \delta}^{\alpha}(M) \leq \mathcal{H}_{A, \delta}^{\alpha}(M)
$$

and therefore " $\geq "$

\section{Estimate of integrals and $\operatorname{dist}\left(L_{x, y}, 0\right)$}

Lemma C.1 (Distance $\operatorname{dist}\left(L_{x, y}, 0\right)$ in terms of $\left.\measuredangle(x, 0, y)\right)$.

Let $x, y \in \mathbb{R}^{n} \backslash\{0\}, x \neq y$ such that $\varepsilon:=\arccos (x \cdot y /(\|x\|\|y\|)) \in(0, \pi)$ and $L_{x, y}$ denote the straight line connecting $x$ and $y$. Then

$$
\operatorname{dist}\left(L_{x, y}, 0\right) \geq \frac{\sin (\varepsilon)}{2} \min \{\|x\|,\|y\|\} .
$$

Proof. Without loss of generality we might assume that $0, x, y \in \mathbb{R}^{2}$. Now we compute the area of the triangle given by $0, x, y$ as

$$
\frac{1}{2} \sin (\varepsilon)\|x\|\|y\|=\frac{1}{2}\|x-y\| \operatorname{dist}\left(L_{x, y}, 0\right)
$$

and obtain

$$
\operatorname{dist}\left(L_{x, y}, 0\right)=\sin (\varepsilon) \frac{\|x\|\|y\|}{\|x-y\|} \geq \sin (\varepsilon) \frac{\|x\|\|y\|}{2 \max \{\|x\|,\|y\|\}}=\frac{\sin (\varepsilon)}{2} \min \{\|x\|,\|y\|\} .
$$

Lemma C.2 (Integral I).

For $y, z>0$ and $p \geq 2$ we have

$$
\int_{0}^{1} \frac{x^{p}}{\left(x^{2}+y^{2}\right)^{p / 2}\left(x^{2}+z^{2}\right)^{p / 2}} d x \leq \frac{\pi}{2^{p}}(z y)^{-(p-1) / 2} .
$$

Proof. We have

$$
\begin{aligned}
& \int_{0}^{1} \frac{x^{p}}{\left(x^{2}+y^{2}\right)^{p / 2}\left(x^{2}+z^{2}\right)^{p / 2}} \mathrm{~d} x=\int_{0}^{1} \frac{x^{p}}{\left(x^{4}+\left(y^{2}+z^{2}\right) x^{2}+y^{2} z^{2}\right)^{p / 2}} \mathrm{~d} x \\
& \stackrel{y^{2}+z^{2} \geq 2 y z}{\leq} \int_{0}^{1} \frac{x^{p}}{\left(x^{4}+2 y z x^{2}+y^{2} z^{2}\right)^{p / 2}} \mathrm{~d} x=\int_{0}^{1} \frac{x^{p}}{\left(x^{2}+y z\right)^{2 p / 2}} \mathrm{~d} x=\int_{0}^{1} \frac{x^{p}}{\left(x^{2}+y z\right)^{p}} \mathrm{~d} x \\
& =\int_{0}^{1} \frac{1}{\left(x+\frac{y z}{x}\right)^{p}} \mathrm{~d} x=\int_{0}^{1} \frac{1}{\left(x+\frac{y z}{x}\right)^{2}} \frac{1}{\left(x+\frac{y z}{x}\right)^{p-2}} \mathrm{~d} x \\
& \stackrel{x+z y / x \geq 2 \sqrt{z y}}{\leq} \int_{0}^{1} \frac{1}{\left(x+\frac{y z}{x}\right)^{2}} \frac{1}{(2 \sqrt{z y})^{p-2}} \mathrm{~d} x \\
& \stackrel{\text { Lemma C. } 3}{=} \frac{1}{2^{p-2}} \frac{1}{(z y)^{p / 2-1}} \frac{1}{2}(\frac{\arctan \left(\frac{1}{\sqrt{z y}}\right)}{\sqrt{z y}}-\underbrace{\frac{1}{1+z y}}_{\geq 0}) \leq \frac{1}{2^{p-2}} \frac{1}{(z y)^{p / 2-1}} \frac{1}{2} \frac{\pi}{2} \frac{1}{\sqrt{z y}} \\
& =\frac{\pi}{2^{p}}(z y)^{-(p-1) / 2}
\end{aligned}
$$




\section{Lemma C.3 (Integral II).}

We have

$$
\int_{0}^{1} \frac{1}{\left(x+\frac{z y}{x}\right)^{2}} d x=\frac{1}{2}\left(\frac{\arctan \left(\frac{1}{\sqrt{z y}}\right)}{\sqrt{z y}}-\frac{1}{1+z y}\right) .
$$

Proof.

$$
\begin{gathered}
{\left[\frac{1}{2}\left(\frac{\arctan \left(\frac{x}{\sqrt{z y}}\right)}{\sqrt{z y}}-\frac{x}{x^{2}+z y}\right)\right]^{\prime}} \\
=\frac{1}{2}(\underbrace{\frac{1}{\sqrt{z y}\left(1+\left(\frac{x}{\sqrt{z y}}\right)^{2}\right)} \frac{1}{\sqrt{z y}}}_{=\frac{1}{z y+x^{2}}}-\frac{1}{x^{2}+z y}+\frac{2 x^{2}}{\left(x^{2}+z y\right)^{2}}) \\
=\frac{x^{2}}{\left(x^{2}+z y\right)^{2}}=\frac{1}{\left(x+\frac{z y}{x}\right)^{2}} .
\end{gathered}
$$

\section{References}

[BK12] Simon Blatt and Sławomir Kolasiński, Sharp Boundedness and Regularizing effects of the integral Menger curvature for submanifolds, to appear in Advances in Mathematics (2012).

[Bla11a] Simon Blatt, A note on integral Menger curvature for curves, Preprint, ETH Zürich, 2011.

[Bla11b] _ The energy spaces of the tangent point energies, Preprint, ETH Zürich, 2011.

[BM70] Leonard M. Blumenthal and Karl Menger, Studies in geometry, W. H. Freeman and Co., San Francisco, Calif., 1970.

[Bra02] Andrea Braides, $\Gamma$-convergence for beginners, Oxford Lecture Series in Mathematics and its Applications, vol. 22, Oxford University Press, Oxford, 2002.

[CKS02] Jason Cantarella, Robert B. Kusner, and John M. Sullivan, On the minimum ropelength of knots and links, Invent. Math. 150 (2002), no. 2, 257286.

[Dud10] James J. Dudziak, Vitushkin's conjecture for removable sets, Universitext, Springer, New York, 2010.

[EG92] Lawrence C. Evans and Ronald F. Gariepy, Measure theory and fine properties of functions, Studies in Advanced Mathematics, CRC Press, Boca Raton, FL, 1992.

[Fal85] Kenneth J. Falconer, The geometry of fractal sets, Cambridge Tracts in Mathematics, vol. 85, Cambridge University Press, Cambridge, 1985. 
[Fol99] Gerald B. Folland, Real analysis, second ed., Pure and Applied Mathematics (New York), John Wiley \& Sons Inc., New York, 1999.

[GdlL03] Oscar Gonzalez and Rafael de la Llave, Existence of ideal knots, J. Knot Theory Ramifications 12 (2003), no. 1, 123-133.

[GM99] Oscar Gonzalez and John H. Maddocks, Global curvature, thickness, and the ideal shapes of knots, Proc. Natl. Acad. Sci. USA 96 (1999), no. 9, 4769-4773 (electronic).

[GMSvdM02] Oscar Gonzalez, John H. Maddocks, Friedemann Schuricht, and Heiko von der Mosel, Global curvature and self-contact of nonlinearly elastic curves and rods, Calc. Var. Partial Differential Equations 14 (2002), no. 1, $29-68$.

[Hah08] Immo Hahlomaa, Menger curvature and rectifiability in metric spaces, Adv. Math. 219 (2008), no. 6, 1894-1915.

[Kol11] Sławomir Kolasiński, Integral Menger curvature for sets of arbitrary dimension and codimension, Preprint, 2011, http://arxiv.org/abs/1011.2008.

[KS11] Sławomir Kolasiński and Marta Szumańska, Minimal Hölder regularity implying finiteness of integral Menger curvature, Preprint, 2011, http://arxiv.org/abs/1111.1141.

[Lég99] Jean-Christophe Léger, Menger curvature and rectifiability, Ann. of Math. (2) 149 (1999), 831-869.

[Lin97] Yong Lin, Menger curvature, singular integrals and analytic capacity, Ann. Acad. Sci. Fenn. Math. Diss. (1997), no. 111, 44, Dissertation, University of Jyväskylä, Jyväskylä, 1997.

[LM01] Yong Lin and Pertti Mattila, Menger curvature and $C^{1}$ regularity of fractals, Proc. Amer. Math. Soc. 129 (2001), no. 6, 1755-1762 (electronic).

[LW09] Gilad Lerman and J. Tyler Whitehouse, High-dimensional Menger-type curvatures. II. d-separation and a menagerie of curvatures, Constr. Approx. 30 (2009), no. 3, 325-360.

[LW11]__ High-dimensional Menger-type curvatures. Part I: Geometric multipoles and multiscale inequalities, Rev. Mat. Iberoam. 27 (2011), no. 2, $493-555$.

[Mat95] Pertti Mattila, Geometry of sets and measures in Euclidean spaces, Cambridge Studies in Advanced Mathematics, vol. 44, Cambridge University Press, Cambridge, 1995.

[Men30] Karl Menger, Untersuchungen über allgemeine Metrik, Math. Ann. 103 (1930), no. 1, 466-501.

[MM88] Miguel Ángel Martín and Pertti Mattila, $k$-dimensional regularity classifications for s-fractals, Trans. Amer. Math. Soc. 305 (1988), no. 1, 293-315. 
[Paj02] Hervé Pajot, Analytic capacity, rectifiability, menger curvature and the cauchy integral, Lecture Notes in Mathematics, vol. 1799, Springer-Verlag, Berlin, 2002.

[Sch11] Sebastian Scholtes, For which positive $p$ is the integral Menger curvature $\mathcal{M}_{p}$ finite for all simple polygons?, Preprint 50, RWTH Aachen University, Institut f. Mathematik, 2011.

[Sch12]__ A characterisation of inner produc spaces by the maximal circumradius of spheres, Preprint 53, RWTH Aachen University, Institut f. Mathematik, 2012.

[SSvdM09] Paweł Strzelecki, Marta Szumańska, and Heiko von der Mosel, A geometric curvature double integral of Menger type for space curves, Ann. Acad. Scient. Fenn. Math. 34 (2009), 195-214.

[SSvdM10] Paweł Strzelecki, Marta Szumańska, and Heiko von der Mosel, Regularizing and self-avoidance effects of integral Menger curvature, Ann. Scuola Norm. Sup. Pisa Cl. Sci. (5) Vol. IX (2010), 1-43.

[SvdM03] Friedemann Schuricht and Heiko von der Mosel, Global curvature for rectifiable loops, Math. Z. 243 (2003), no. 1, 37-77.

[SvdM04] _ Characterization of ideal knots, Calc. Var. Partial Differential Equations 19 (2004), no. 3, 281-305.

[SvdM05] Paweł Strzelecki and Heiko von der Mosel, On a mathematical model for thick surfaces, Physical and Numerical Models in Knot Theory (Calvo, Millett, Rawdon, and Stasiak, eds.), Ser. Knots Everything, vol. 36, World Sci. Publ., Singapore, 2005, pp. 547-564.

[SvdM06]__ Global curvature for surfaces and area minimization under a thickness constraint, Calc. Var. Partial Differential Equations 25 (2006), no. 4, 431-467.

[SvdM07] _ On rectifiable curves with $L^{p}$-bounds on global curvature: selfavoidance, regularity, and minimizing knots, Math. Z. 257 (2007), no. 1, $107-130$.

[SvdM11] _ Integral Menger curvature for surfaces, Adv. Math. 226 (2011), no. 3, 2233-2304.

[SvdM12a] _ Tangent-point repulsive potentials for a class of non-smooth mdimensional sets in $\mathbb{R}^{n}$. Part I: Smoothing and self-avoidance effects, to appear in J. Geometric Analysis (2012), http://dx.doi.org/10.1007/s12220011-9275-z.

[SvdM12b] _ Tangent-point self-avoidance energies for curves, to appear in J. Knot Theory Ramifications 21 (2012), no. 2, http://dx.doi.org/10.1142/S0218216511009960. 
Sebastian Scholtes

Institut für Mathematik

RWTH Aachen University

Templergraben 55

D-52062 Aachen, Germany

sebastian.scholtes@rwth-aachen.de 


\section{Reports des Instituts für Mathematik der RWTH-Aachen}

[1] Bemelmans J.: Die Vorlesung "Figur und Rotation der Himmelskörper" von F. Hausdorff, WS 1895/96, Universität Leipzig, S 20, März 2005

[2] Wagner A.: Optimal Shape Problems for Eigenvalues, S 30, März 2005

[3] Hildebrandt S. and von der Mosel H.: Conformal representation of surfaces, and Plateau's problem for Cartan functionals, S 43, Juli 2005

[4] Reiter P.: All curves in a $C^{1}$-neighbourhood of a given embedded curve are isotopic, S 8, Oktober 2005

[5] Maier-Paape S., Mischaikow K. and Wanner T.: Structure of the Attractor of the Cahn-Hilliard Equation, S 68, Oktober 2005

[6] Strzelecki P. and von der Mosel H.: On rectifiable curves with $L^{p}$ bounds on global curvature: Self-avoidance, regularity, and minimizing knots, S 35, Dezember 2005

[7] Bandle C. and Wagner A.: Optimization problems for weighted Sobolev constants, S 23, Dezember 2005

[8] Bandle C. and Wagner A.: Sobolev Constants in Disconnected Domains, S 9, Januar 2006

[9] McKenna P.J. and Reichel W.: A priori bounds for semilinear equations and a new class of critical exponents for Lipschitz domains, S 25, Mai 2006

[10] Bandle C., Below J. v. and Reichel W.: Positivity and anti-maximum principles for elliptic operators with mixed boundary conditions, S 32, Mai 2006

[11] Kyed M.: Travelling Wave Solutions of the Heat Equation in Three Dimensional Cylinders with Non-Linear Dissipation on the Boundary, S 24, Juli 2006

[12] Blatt S. and Reiter P.: Does Finite Knot Energy Lead To Differentiability?, S 30, September 2006

[13] Grunau H.-C., Ould Ahmedou M. and Reichel W.: The Paneitz equation in hyperbolic space, S 22, September 2006

[14] Maier-Paape S., Miller U.,Mischaikow K. and Wanner T.: Rigorous Numerics for the Cahn-Hilliard Equation on the Unit Square, S 67, Oktober 2006

[15] von der Mosel H. and Winklmann S.: On weakly harmonic maps from Finsler to Riemannian manifolds, S 43, November 2006

[16] Hildebrandt S., Maddocks J. H. and von der Mosel H.: Obstacle problems for elastic rods, S 21, Januar 2007

[17] Galdi P. Giovanni: Some Mathematical Properties of the Steady-State Navier-Stokes Problem Past a ThreeDimensional Obstacle, S 86, Mai 2007

[18] Winter N.: $W^{2, p}$ and $W^{1, p}$-estimates at the boundary for solutions of fully nonlinear, uniformly elliptic equations, S 34, July 2007

[19] Strzelecki P., Szumańska M. and von der Mosel H.: A geometric curvature double integral of Menger type for space curves, S 20, September 2007

[20] Bandle C. and Wagner A.: Optimization problems for an energy functional with mass constraint revisited, S 20, März 2008

[21] Reiter P., Felix D., von der Mosel H. and Alt W.: Energetics and dynamics of global integrals modeling interaction between stiff filaments, S 38, April 2008

[22] Belloni M. and Wagner A.: The $\infty$ Eigenvalue Problem from a Variational Point of View, S 18, Mai 2008

[23] Galdi P. Giovanni and Kyed M.: Steady Flow of a Navier-Stokes Liquid Past an Elastic Body, S 28, Mai 2008

[24] Hildebrandt S. and von der Mosel H.: Conformal mapping of multiply connected Riemann domains by a variational approach, S 50, Juli 2008

[25] Blatt S.: On the Blow-Up Limit for the Radially Symmetric Willmore Flow, S 23, Juli 2008 
[26] Müller F. and Schikorra A.: Boundary regularity via Uhlenbeck-Rivière decomposition, S 20, Juli 2008

[27] Blatt S.: A Lower Bound for the Gromov Distortion of Knotted Submanifolds, S 26, August 2008

[28] Blatt S.: Chord-Arc Constants for Submanifolds of Arbitrary Codimension, S 35, November 2008

[29] Strzelecki P., Szumańska M. and von der Mosel H.: Regularizing and self-avoidance effects of integral Menger curvature, S 33, November 2008

[30] Gerlach H. and von der Mosel H.: Yin-Yang-Kurven lösen ein Packungsproblem, S 4, Dezember 2008

[31] Buttazzo G. and Wagner A.: On some Rescaled Shape Optimization Problems, S 17, März 2009

[32] Gerlach H. and von der Mosel H.: What are the longest ropes on the unit sphere?, S 50, März 2009

[33] Schikorra A.: A Remark on Gauge Transformations and the Moving Frame Method, S 17, Juni 2009

[34] Blatt S.: Note on Continuously Differentiable Isotopies, S 18, August 2009

[35] Knappmann K.: Die zweite Gebietsvariation für die gebeulte Platte, S 29, Oktober 2009

[36] Strzelecki P. and von der Mosel H.: Integral Menger curvature for surfaces, S 64, November 2009

[37] Maier-Paape S., Imkeller P.: Investor Psychology Models, S 30, November 2009

[38] Scholtes S.: Elastic Catenoids, S 23, Dezember 2009

[39] Bemelmans J., Galdi G.P. and Kyed M.: On the Steady Motion of an Elastic Body Moving Freely in a Navier-Stokes Liquid under the Action of a Constant Body Force, S 67, Dezember 2009

[40] Galdi G.P. and Kyed M.: Steady-State Navier-Stokes Flows Past a Rotating Body: Leray Solutions are Physically Reasonable, S 25, Dezember 2009

[41] Galdi G.P. and Kyed M.: Steady-State Navier-Stokes Flows Around a Rotating Body: Leray Solutions are Physically Reasonable, S 15, Dezember 2009

[42] Bemelmans J., Galdi G.P. and Kyed M.: Fluid Flows Around Floating Bodies, I: The Hydrostatic Case, S 19, Dezember 2009

[43] Schikorra A.: Regularity of n/2-harmonic maps into spheres, S 91, März 2010

[44] Gerlach H. and von der Mosel H.: On sphere-filling ropes, S 15, März 2010

[45] Strzelecki P. and von der Mosel H.: Tangent-point self-avoidance energies for curves, S 23, Juni 2010

[46] Schikorra A.: Regularity of n/2-harmonic maps into spheres (short), S 36, Juni 2010

[47] Schikorra A.: A Note on Regularity for the $n$-dimensional H-System assuming logarithmic higher Integrability, S 30, Dezember 2010

[48] Bemelmans J.: Über die Integration der Parabel, die Entdeckung der Kegelschnitte und die Parabel als literarische Figur, S 14, Januar 2011

[49] Strzelecki P. and von der Mosel H.: Tangent-point repulsive potentials for a class of non-smooth $m$-dimensional sets in $\mathbb{R}^{n}$. Part I: Smoothing and self-avoidance effects, S 47, Februar 2011

[50] Scholtes S.: For which positive $p$ is the integral Menger curvature $\mathcal{M}_{p}$ finite for all simple polygons, S 9, November 2011

[51] Bemelmans J., Galdi G. P. and Kyed M.: Fluid Flows Around Rigid Bodies, I: The Hydrostatic Case, S 32, Dezember 2011

[52] Scholtes S.: Tangency properties of sets with finite geometric curvature energies, S 39, February 2012

[53] Scholtes S.: A characterisation of inner product spaces by the maximal circumradius of spheres, S 8, February 2012 